\title{
Cell-type Dependent Alzheimer's Disease Phenotypes: Probing the Biology of Selective Neuronal Vulnerability
}

\section{Citation}

Muratore, C. R., C. Zhou, M. Liao, M. A. Fernandez, W. M. Taylor, V. N. Lagomarsino, R. V. Pearse, et al. 2017. "Cell-type Dependent Alzheimer's Disease Phenotypes: Probing the Biology of Selective Neuronal Vulnerability." Stem Cell Reports 9 (6): 1868-1884. doi:10.1016/ j.stemcr.2017.10.015. http://dx.doi.org/10.1016/j.stemcr.2017.10.015.

\section{Published Version}

doi:10.1016/j.stemcr.2017.10.015

\section{Permanent link}

http://nrs.harvard.edu/urn-3:HUL.InstRepos:34868998

\section{Terms of Use}

This article was downloaded from Harvard University's DASH repository, and is made available under the terms and conditions applicable to Other Posted Material, as set forth at http:// nrs.harvard.edu/urn-3:HUL.InstRepos:dash.current.terms-of-use\#LAA

\section{Share Your Story}

The Harvard community has made this article openly available.

Please share how this access benefits you. Submit a story.

Accessibility 


\title{
Cell-type Dependent Alzheimer's Disease Phenotypes: Probing the Biology of Selective Neuronal Vulnerability
}

\author{
Christina R. Muratore, ${ }^{1}$ Constance Zhou, ${ }^{1}$ Meichen Liao, ${ }^{1}$ Marty A. Fernandez, ${ }^{1}$ Walter M. Taylor, ${ }^{1}$ \\ Valentina N. Lagomarsino, ${ }^{1}$ Richard V. Pearse II, ${ }^{1}$ Heather C. Rice, ${ }^{1}$ Joseph M. Negri, ${ }^{1}$ Amy He, ${ }^{1}$ \\ Priya Srikanth, ${ }^{1}$ Dana G. Callahan, ${ }^{1}$ Taehwan Shin, ${ }^{1}$ Monica Zhou, ${ }^{4}$ David A. Bennett, ${ }^{5}$ Scott Noggle, ${ }^{4}$ \\ J. Christopher Love, ${ }^{2,3}$ Dennis J. Selkoe, ${ }^{1}$ and Tracy L. Young-Pearse ${ }^{1, *}$ \\ ${ }^{1}$ Ann Romney Center for Neurologic Diseases, Brigham and Women's Hospital and Harvard Medical School, Boston, MA 02115, USA \\ ${ }^{2}$ Koch Institute for Integrative Cancer Research, Massachusetts Institute of Technology, Cambridge, MA 02139, USA \\ ${ }^{3}$ Department of Chemical Engineering, Massachusetts Institute of Technology, Cambridge, MA 02139, USA \\ ${ }^{4}$ The New York Stem Cell Foundation Research Institute, New York, NY 10019, USA \\ ${ }^{5}$ Rush Alzheimer's Disease Center, Rush University Medical Center, Chicago, IL 60612, USA \\ *Correspondence: tyoung@rics.bwh.harvard.edu \\ https://doi.org/10.1016/j.stemcr.2017.10.015
}

\section{SUMMARY}

Alzheimer's disease (AD) induces memory and cognitive impairment in the absence of motor and sensory deficits during its early and middle course. A major unresolved question is the basis for this selective neuronal vulnerability. A $\beta$, which plays a central role in $\mathrm{AD}$ pathogenesis, is generated throughout the brain, yet some regions outside of the limbic and cerebral cortices are relatively spared from $A \beta$ plaque deposition and synapse loss. Here, we examine neurons derived from iPSCs of patients harboring an amyloid precursor protein mutation to quantify AD-relevant phenotypes following directed differentiation to rostral fates of the brain (vulnerable) and caudal fates (relatively spared) in AD. We find that both the generation of $\mathrm{A} \beta$ and the responsiveness of TAU to A $\beta$ are affected by neuronal cell type, with rostral neurons being more sensitive than caudal neurons. Thus, cell-autonomous factors may in part dictate the pattern of selective regional vulnerability in human neurons in $\mathrm{AD}$.

\section{INTRODUCTION}

Alzheimer's disease $(\mathrm{AD})$ is a prevalent neurodegenerative disorder characterized by extracellular plaques composed of amyloid $\beta$-protein $(\mathrm{A} \beta)$ and intraneuronal tangles consisting of altered forms of TAU. Importantly, distinct brain regions are differentially susceptible to plaque accumulation and TAU-associated neurodegeneration in $\mathrm{AD}$, with the limbic and association areas being primarily affected and the basal ganglia, hindbrain, and spinal cord being initially spared. A central unanswered question in $\mathrm{AD}$, as in other neurodegenerative diseases, is the basis for this selective vulnerability: why neurons in the basal ganglia, cerebellum, brain stem, and spinal cord are able to function effectively to allow relatively unperturbed motor, sensory, and autonomic function, while the hippocampus, amygdala, and cerebral cortex are progressively devastated.

At least three broad mechanisms could potentially underlie the differential regional vulnerability observed in neurodegenerative diseases: (1) altered production (and perhaps extracellular release) of toxic species between neurons in different regions; (2) varying clearance of toxic species among brain regions; and/or (3) distinct sensitivities to synaptotoxic species resulting from intrinsic molecular differences between neuronal fates. Each of these mechanisms may contribute to differential neuronal dysfunction and loss in $\mathrm{AD}$. Here we focused on the potential contribu- tions of intrinsic (cell-autonomous) differences among neuronal subtypes that could affect both the generation of, and the responses to, cytotoxic species. Given the difficulty of acquiring living human neurons directly from patient brain, we took advantage of human induced pluripotent stem cell (iPSC) technology to generate neuronal cultures of differing regional fates. While we will discuss the caveats to this in vitro model system, it allows one to take a well-controlled, reductionist approach to address whether and how intrinsically encoded differences between neuronal fates mediate selective vulnerability in AD.

Early-onset, familial Alzheimer's disease (fAD) accounts for a small minority of all cases of AD. However, the study of $\mathrm{fAD}$ patients has revealed important aspects of the mechanisms underlying all types of $\mathrm{AD}$. Early-onset $\mathrm{fAD}$ is caused by dominant, highly penetrant mutations in either presenilin (PSEN) or amyloid precursor protein (APP) (reviewed in Bertram et al., 2010). PSEN encodes the catalytic site of $\gamma$-secretase. APP is a single transmembrane domain protein that can be cleaved by either an $\alpha$ - or $\beta$-secretase, resulting in the shedding of large extracellular portions of APP termed sAPP $\alpha$ or SAPP $\beta$, respectively. Sequential cleavage by $\beta$-secretase followed by the PSEN $/ \gamma$-secretase complex produces $A \beta$ peptides of various lengths, most commonly 40, 42, and 38 amino acids long. fAD mutations in both APP and PSEN have been shown to favor the production of the more aggregation-prone $A \beta 42$ over $A \beta 40$, suggesting that an altered ratio of $A \beta 42$ and $A \beta 40$ 
A

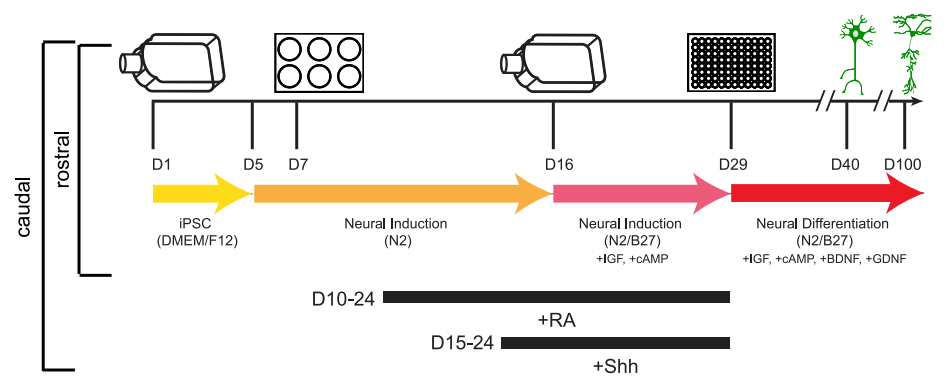

B

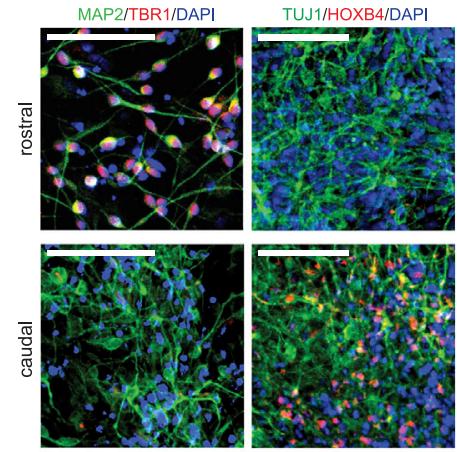

E

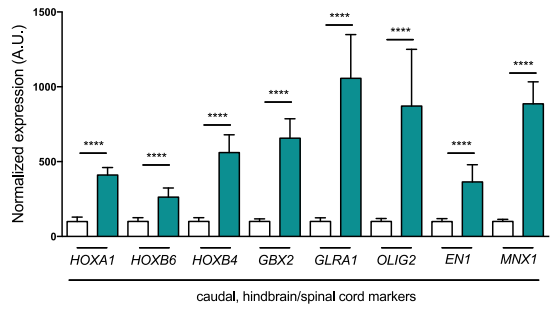

G
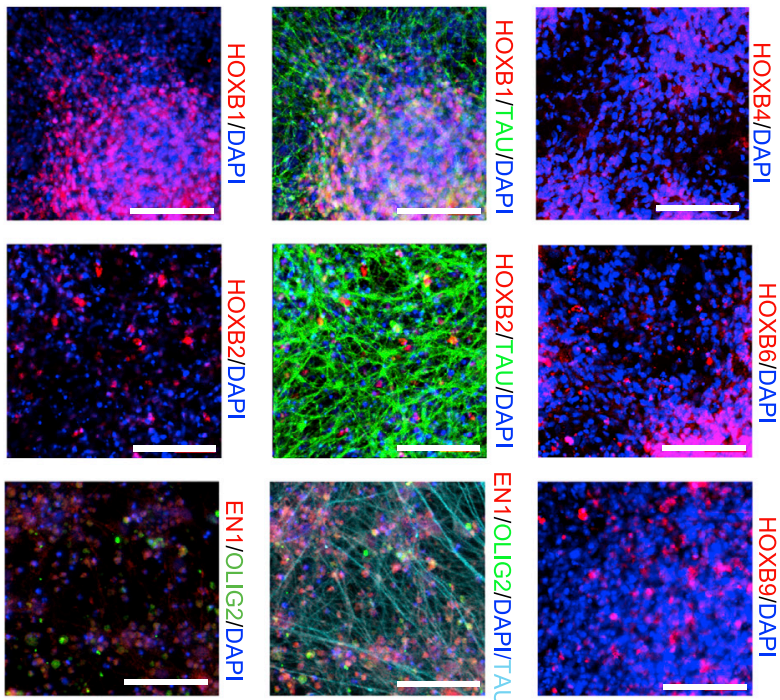

H

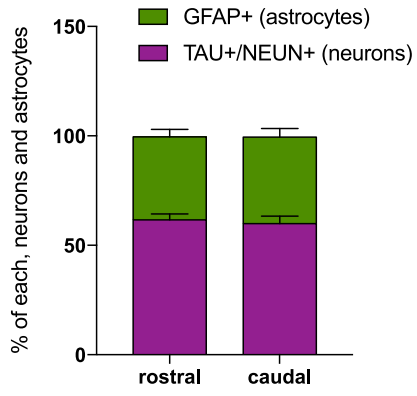

I

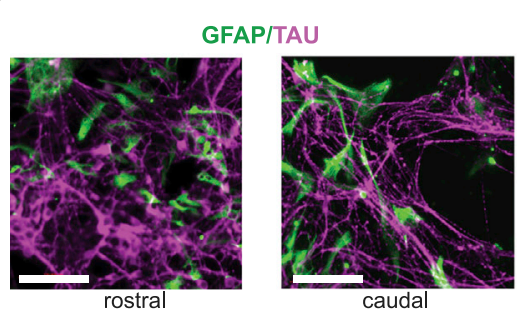


contributes to the formation of the $A \beta$ plaques seen in the brains of AD patients (Bentahir et al., 2006; Scheuner et al., 1996).

Due to recent progress in iPSC technology, human neurons differentiated from iPSC lines can be used to model neurological diseases. We previously described the generation and detailed characterization of iPSC lines from two carriers of an fAD mutation, the "London" mutation, in APP (APPV717I) (Muratore et al., 2014a). By comparing neurons differentiated from fAD APPV717I iPSC lines with controls, we observed significant changes in APP processing and the levels of phosphorylated and total TAU (Muratore et al., 2014a). That study was performed entirely in neurons differentiated to a forebrain fates of the cerebral cortex. However, iPSC-derived neurons can be efficiently patterned to different neuronal subtypes. Here, we directly compare control and APPV717I iPSCs differentiated to rostral, cortical fates with caudal neural fates of the hindbrain and spinal cord. We use this culture system to probe key questions regarding how neuronal cell type affects processing of APP by $\alpha$-, $\beta$-, and $\gamma$-secretases, as well as the responsiveness of different neuronal subtypes to $A \beta$. We find that caudal neurons differ from rostral neurons in both their generation of and responsiveness to $\mathrm{A} \beta$ species. APPV717I neurons directed to caudal neuronal fates generate $A \beta$ with a lower $42: 40$ ratio and higher 38:42 ratio than rostral telencephalic neurons. Further, we show that APPV717I neurons express higher levels of total and phospho-TAU proteins relative to control neurons when directed to a rostral neuronal fate, but not when directed to a caudal neuronal fate. Finally, we demonstrate that neurons of these different cell fates respond differentially to soluble extracts of clinically and neuropathologically typical "sporadic" late-onset AD (LOAD) brains. These AD brain extracts induce an elevation in the phosphorylation of TAU in forebrain neurons, and this is dependent upon the $A \beta$ present in these extracts. However, when exposed to the same $\mathrm{AD}$ extracts, TAU phosphorylation is not affected in neurons directed to caudal fates. Taken together, these results suggest that both APP processing and TAU proteostasis are differentially altered between neuronal subtypes that are relatively vulnerable or resistant to $\mathrm{AD}$.

\section{RESULTS}

Directed Differentiation to Alternate Neuronal Fates Human iPSCs from a father and daughter each expressing the fAD APPV717I mutation were previously generated and characterized by our lab (Muratore et al., 2014a). Here, iPSC lines from both fAD APPV717I subjects and subjects who do not harbor fAD mutations were directed to neuronal fates using an embryoid aggregate protocol, as described previously (Muratore et al., 2014b, 2014a). In the absence of exogenously administered patterning factors, the default pathway of this differentiation protocol is to generate forebrain neurons of cortical fates. To direct the differentiation of these cells to caudal neuronal fates, the embryoid aggregate protocol was modified to include treatment with retinoic acid (RA) and Sonic hedgehog (Shh) (or a Shh agonist, purmorphamine [purm]). RA and $\mathrm{Shh} /$ purm were added to cultures at the neural progenitor stage between days 10 and 24 and 15 and 24 of differentiation, respectively (Hu and Zhang, 2009) (Figure 1A). At day 40 of differentiation, cultures were fixed and immunostained for cell fate markers (Figure 1B). Caudally directed cultures expressed qualitatively similar numbers of MAP2+ and TUJ1+ cells as rostral cultures (Figure 1B). For a more quantitative comparison between rostral and caudal cultures, RNA was harvested to analyze gene expression using a custom NanoString CodeSet (Figures 1C-1E). Markers of general neuronal fate, such as MAP2 and TUJ1, were unchanged whether differentiation proceeded in the absence or presence of RA/Shh (Figure 1C). However, with RA/Shh treatment, markers of neuronal fates of the forebrain (cerebral cortex), such as TBR1, FEZF2, and

Figure 1. Differentiation of Human iPSCs to Rostral and Caudal Neural Fates

(A) Schematic of differentiation to neuronal fates showing window of morphogen treatment. Retinoic acid (RA) and Sonic hedgehog (Shh) treatments are indicated by black bars.

(B) Immunostaining of day 40 iPSCs differentiated to rostral and caudal neuronal fates. Scale bars, $100 \mu \mathrm{m}$.

(C-E) NanoString analysis of expression of a subset of 150 genes analyzed in rostral and caudal cultures differentiated for 40 days. Rostral and caudal neurons are indicated by white and teal bars, respectively. Data in $(C)-(E)$ are derived from six lines over nine independent rounds of differentiation. Rostral neurons, $n=44$; caudal neurons, $n=32$. Data are represented as mean \pm SEM. For each gene, a Student's $t$ test was performed, $* * * * p<0.0001$. Raw NanoString data were normalized to all genes in the custom CodeSet.

(F) Hox gene expression was measured via RNA sequencing, and the mean expression level ( $n=4$ for rostral, $n=4$ for caudal) is shown in a heatmap.

(G) Representative images of immunostaining for listed caudal markers are shown. Scale bars, $50 \mu \mathrm{m}$.

( $\mathrm{H}$ and I) Quantification of the percentage of neurons and astrocytes in rostral and caudal cultures (day 100) determined by immunostaining. Data are derived from three lines over three independent rounds of differentiation. Rostral neurons, $n=11 ;$ caudal neurons, $n=9$. Scale bars, $100 \mu \mathrm{m}$.

See also Figure S1. 
A

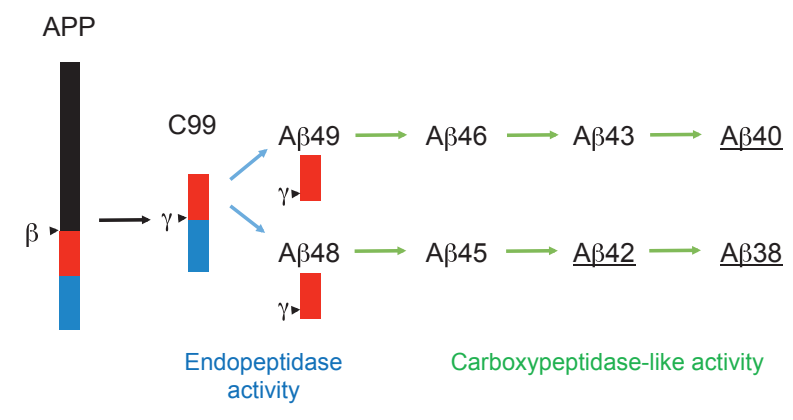

B

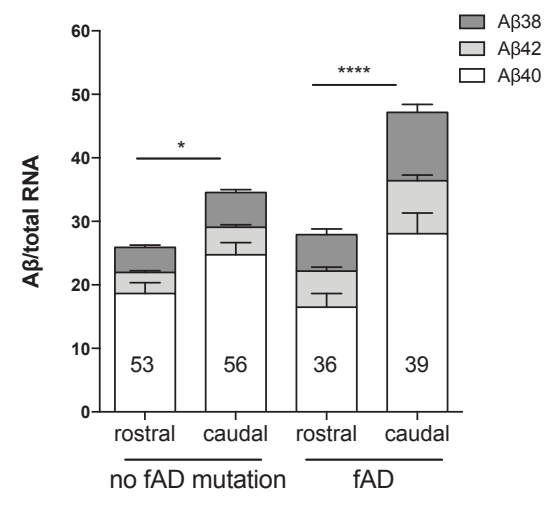

C

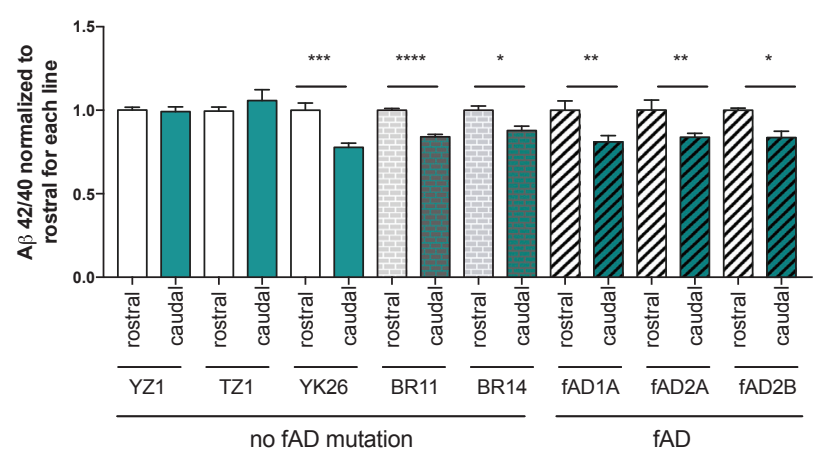

$\mathbf{E}$

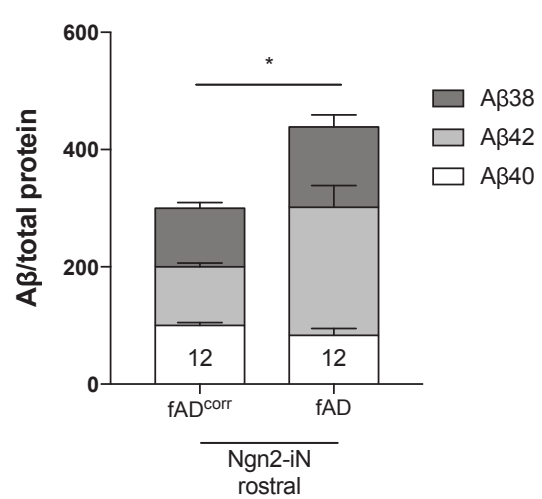

$\mathbf{F}$

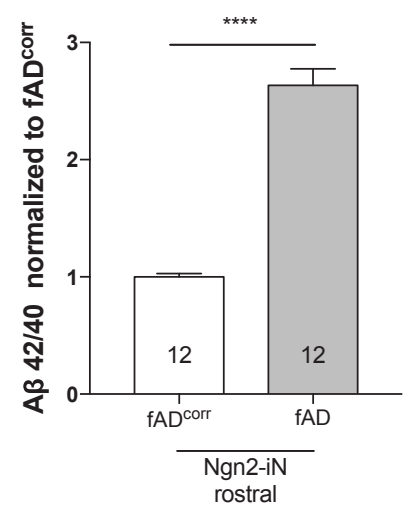

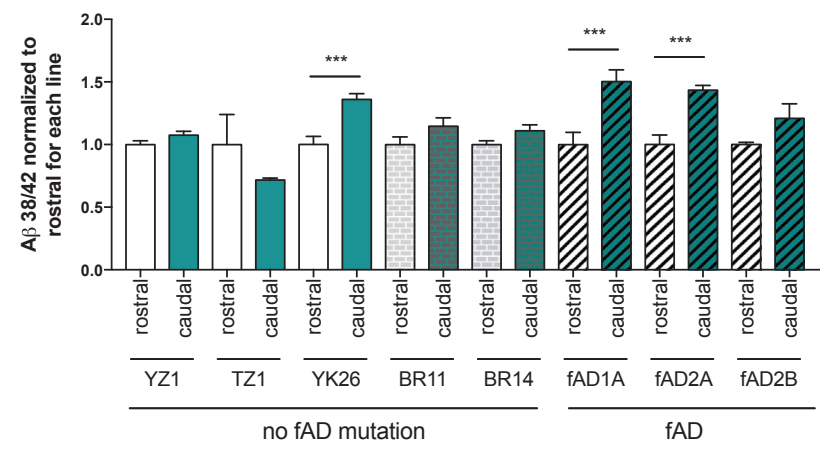

G

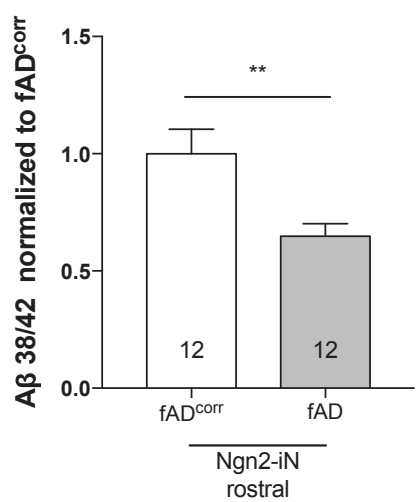

Figure 2. APP is Differentially Cleaved in Rostral Versus Caudal Neural Cultures

(A) Schematic of cleavage steps of APP to generate A $\beta$ of differing lengths. Black box, SAPP $\beta$; red box, A $\beta$; blue box, APP intracellular domain, underlined species were measured by MSD triplex ELISA. Green arrows denote the two major processing lines for $\gamma$-secretase. However, a small degree of crossover between pathways can occur as minor events at any step.

(B-G) Following an embryoid aggregate differentiation of non-fAD mutation and APPV717I (fAD) lines, conditioned media from day 40 rostral and caudal cultures were analyzed. $A \beta 38, A \beta 40$, and $A \beta 42$ were measured by $A \beta$ triplex ELISA. (B) Levels of $A \beta 38$, $A \beta 40$, and $A \beta 42$ are shown normalized to total RNA extracted from the corresponding lysate. The ratios of $A \beta 42: 40$ (C) and 38:42 (D) are shown normalized to rostral control. The " $\mathrm{n}$ " for each rostral/caudal pair is the following: YZ1, 38/38; TZ1, 9/9; YK26, 6/9; BR11, 11/12; BR14, 8/8; fAD1a, 14/14; fAD 2A, 8/6; and fAD 2B, 14/19. There are 12 independent rounds of differentiation represented. Rostral and caudal cultures are indicated by white and teal bars, respectively. Data in (E)-(G) are derived from day $28 \mathrm{Ngn} 2$-iN cultures. Conditioned media from both $f A D$ and $A A D^{\text {corr }}$ were analyzed for $A \beta 38, A \beta 40$, and $A \beta 42$ by $A \beta$ triplex ELISA. (E) Levels of $A \beta 38, A \beta 40$, and $A \beta 42$ are shown normalized to total protein. Each individual $f A D A \beta$ value is normalized to $100 \%$ of its paired $f A D^{\text {corr }} A \beta$ value for that differentiation. The ratios of $A \beta 42: 40(F)$

(legend continued on next page) 
CTIP2, were downregulated (Figures 1B, left column and 1D), while markers of more caudal (hindbrain/spinal cord) fates were upregulated (Figures $1 \mathrm{~B}$ right column and $1 \mathrm{E})$. For example, $H B 9$ is a homeobox gene expressed selectively by lower motor neurons, and HOXB4 is expressed in the spinal cord and hindbrain with no expression in the midbrain and forebrain. $H O X A 1, H O X B 6$, and EN1 also were upregulated in the caudally directed cultures, consistent with the presence of neurons of hindbrain/spinal cord fates. In addition, we examined the relative expression levels of all HOX genes through RNA sequencing of rostral and caudal cultures (Figure 1F). At the RNA level, HOXB9 was present at the highest level in caudal cultures, followed by $H O X B 8$ and HOXB3. To complement this dataset and further define caudal cultures, we performed immunostaining for some of the targets that were highly expressed in caudal cultures at the RNA level (Figure 1G). These results are in agreement with multiple studies showing that stimulating the Shh pathway along with RA in neural progenitors directs cells to more caudal fates of the hindbrain and spinal cord (Hu and Zhang, 2009; Lee et al., 2007; Li et al., 2008). As described previously (Liao et al., 2016; Muratore et al., 2014b), at day 40 rostral cultures are primarily neuronal (>95\% MAP2 positive), with a minor proportion of progenitor cells that ultimately give rise to cells expressing astrocyte markers. Analyses in portions of this study occur at day 100 of differentiation, when significant numbers of astrocytes are present. Therefore, we quantified the percentage of neurons and astrocytes in rostral and caudal cultures at this time point across several iPSC lines and differentiation rounds (Figures $1 \mathrm{H}$ and 1I). Both rostral and caudal cultures contained cells expressing neuronal markers (TAU+/NeuN+; $\sim 60 \%$ ) and astrocyte markers (GFAP+; $\sim 40 \%$ ). As reported previously by us and others, different lines and differentiation rounds can show variability in the efficiency of differentiation when using an embryoid body (EB)-based protocol. To be included in subsequent analyses, rostral and caudal cultures needed to pass multiple levels of quality control, as outlined in Figure S1.

\section{APP Is Differentially Processed in Human iPSCs}

Directed to Caudal versus Rostral Neuronal Fates

Early-onset fAD is caused by dominantly inherited and highly penetrant mutations in the genes encoding APP and PSEN. Hundreds of these mutations have been identified, and virtually all mutations investigated have been shown to elevate the $42: 40$ ratio by increasing levels of $\mathrm{A} \beta 42$ and/or decreasing levels of $\mathrm{A} \beta 40$ (Bentahir et al.,
2006; Scheuner et al., 1996). The two additional amino acids in $A \beta 42$ are hydrophobic and make $A \beta 42$ more prone to aggregation. The lengths of $A \beta$ peptides generated are dependent upon both the site of initial endopeptidase (epsilon) cleavage and the carboxypeptidase-like progressive cleavages, both of which can be affected by the $\gamma$-secretase complex (Figure $2 \mathrm{~A}$ ). To better understand the $\mathrm{A} \beta$ composition in our cultures, we assayed 48-hr conditioned medium using a triplex $A \beta$ ELISA for $A \beta 38, A \beta 40$, and $A \beta 42$. Caudally directed neurons showed an overall increase in $A \beta$ production (Figure 2B) compared with forebrain cultures, and this increase in $\mathrm{A} \beta$ was more pronounced in $\mathrm{fAD}$ caudal cultures $(\sim 40 \%)$ than in control caudal cultures ( $25 \%)$. More specifically, fAD neurons directed to caudal fates showed a greater increase in both $\mathrm{A} \beta 38(\sim 88 \%)$ and $\mathrm{A} \beta 40$ $(\sim 70 \%)$ than in $\mathrm{A} \beta 42(\sim 46 \%)$ compared with neurons directed to a rostral fate. These changes in the pattern of $\mathrm{A} \beta$ generation suggest that both the endopeptidase and carboxypeptidase activities of $\gamma$-secretase are altered as a function of regional neuronal identity. We and others have previously shown that fAD forebrain neurons secrete A $\beta$ with an elevated 42:40 ratio, compared with control forebrain neurons (Israel et al., 2012; Koch et al., 2012; Kondo et al., 2013; Moore et al., 2015; Muratore et al., 2014a). Interestingly, when fAD neurons are directed to caudal fates, the A $\beta 42: 40$ ratio is significantly decreased by approximately $15 \%$ relative to the same iPSCs directed in parallel to forebrain fates (Figures 2C and S2A). In control (no fAD mutation) cultures, the $\mathrm{A} \beta 42: 40$ ratio was not decreased in caudal cultures in one genetic background (YZ1 and TZ1), but was reduced in five others (Figure 2C). Moreover, caudally directed neurons in three genetic backgrounds showed a significant elevation in the ratio of A $\beta 38: 42$ relative to their rostral paired cultures, and a similar trend observed in three other genetic backgrounds (Figures $2 \mathrm{D}$ and $\mathrm{S} 2 \mathrm{~B}$ ). The increases in $\mathrm{A} \beta 38$ and $\mathrm{A} \beta 40$ drive the observed changes in the peptide ratios (Figure $2 \mathrm{~B}$ ).

Our fAD iPSC lines are derived from two individuals, a father and daughter. To examine the effects of the same fAD mutation in other genetic backgrounds, we used lentivirus to express APPV717I in day 40 rostral and caudal cultures from two control lines (BR11 and BR01). As expected, APP717I expression dramatically elevated the A $\beta 42: 40$ ratio in both rostral and caudal cultures, relative to mock transduced cultures. However, the effect of the APP717I mutation on $A \beta 42: 40$ ratio was greater in rostral relative to control caudal cultures, with a significant reduction in $\mathrm{A} \beta 42: 40$ ratio and elevation of $\mathrm{A} \beta 38: 42$ ratio in caudal

and 38:42 (G) are shown normalized to $f A D^{\text {corr }}$. The " $n$ " for each dataset is shown within the bar graph column. There are four independent rounds of differentiation represented. For each comparison, a Student's t test was performed, ${ }^{*} p<0.05,{ }^{* *} p<0.01,{ }^{* * *} p<0.001,{ }^{* * * *} p<$ 0.0001 . Data are represented as mean \pm SEM.

See also Figures S2-S4. 
A

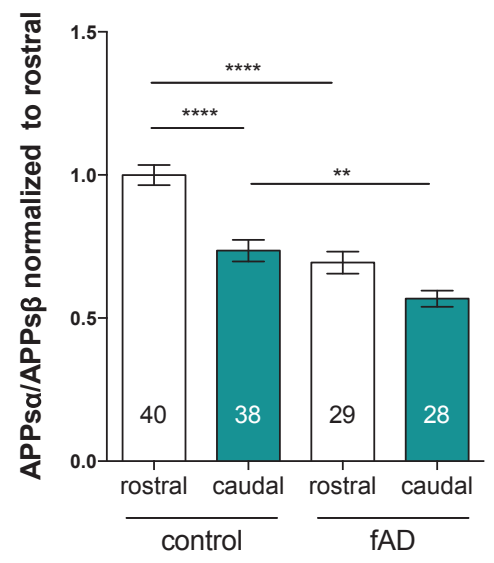

D

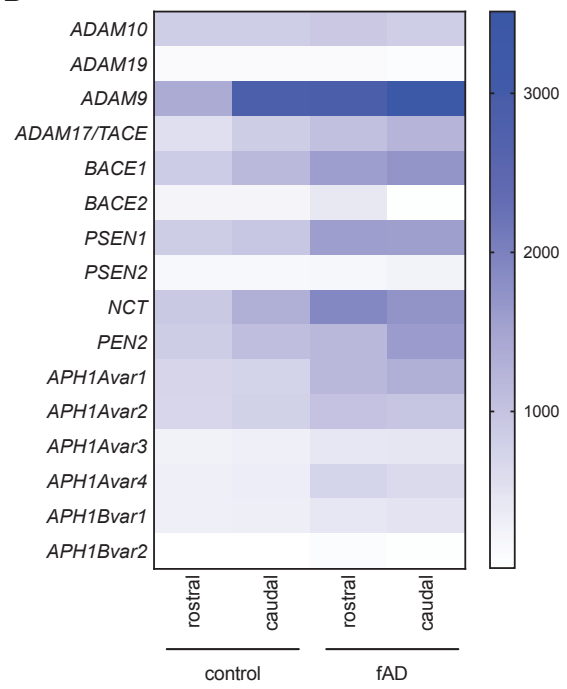

G

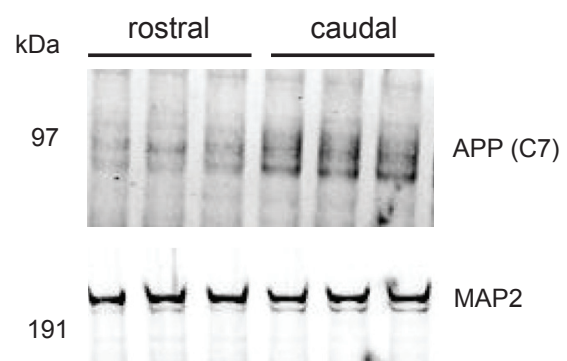

B

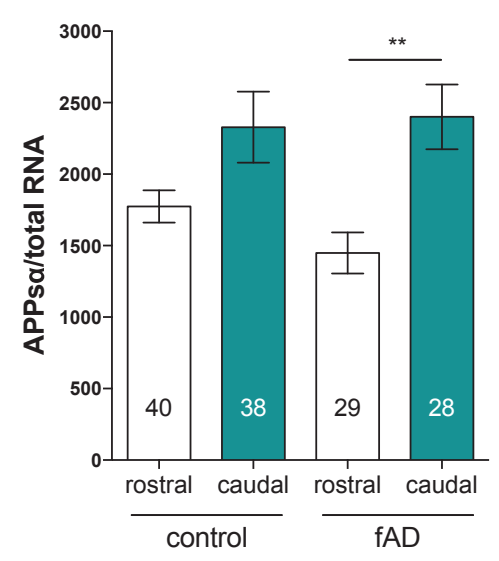

E

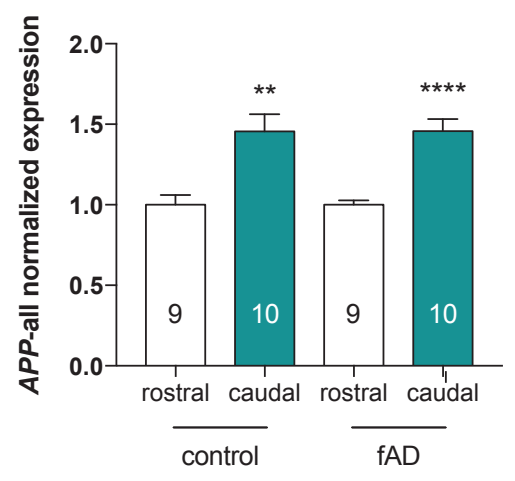

H

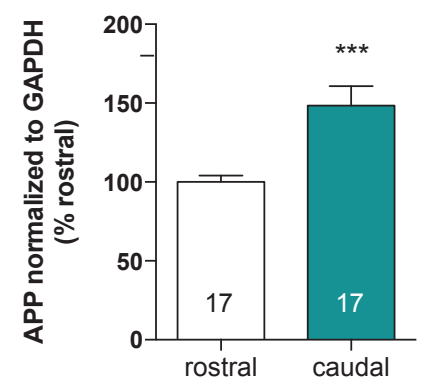

C

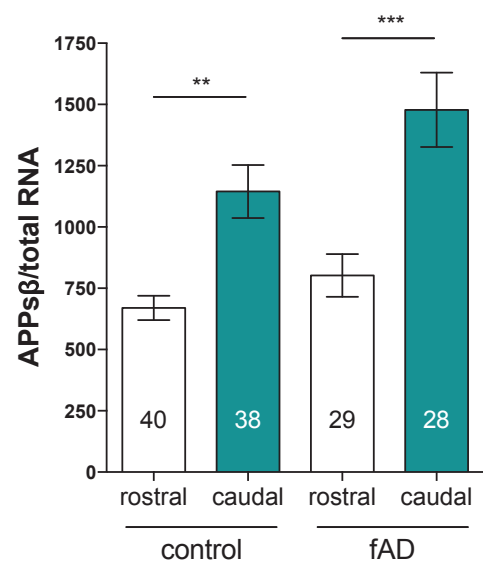

$\mathbf{F}$

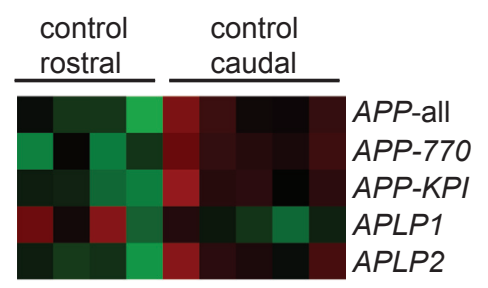

fAD rostral fAD caudal

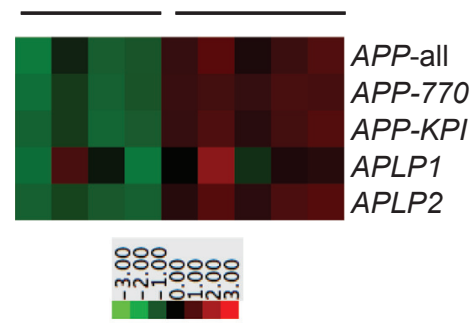

39

GAPDH

Figure 3. APP Expression Is Elevated in Caudally Directed Neural Cultures Relative to Rostral Cultures

$(A-C)$ Secretion of SAPP $\alpha$ and SAPP $\beta$ were measured by duplex ELISA. Ratios of SAPP $\alpha /$ SAPP $\alpha \beta$ are shown (normalized to rostral control) (A), as well as SAPP $\alpha$ normalized to total RNA (B) and SAPP $\beta$ normalized to total RNA (C). Rostral and caudal cultures are indicated by white 
versus rostral APPV717I-expressing cultures (Figures S3AS3C). We confirmed overexpression of APPV717I with western blot analysis (Figure S3D). Interestingly, although this method is overexpressing APP in both rostral and caudal cultures (Figure S3D), caudal cultures showed a significant increase in total $A \beta$ relative to rostral cultures transduced in parallel (Figure S3A), similar to the result observed in the endogenous expression models.

Prior to $\gamma$-secretase cleavage, APP must first be cleaved by $\beta$-secretase in order for $A \beta$ generation to occur. This cleavage event generates SAPP $\beta$, the shed ectodomain product of APP. Cleavage instead by $\alpha$-secretase generates sAPP $\alpha$, which precludes $A \beta$ generation. To investigate whether $\alpha$ and $\beta$-cleavages of APP also were affected by neuronal fate, we measured secretion of SAPP $\alpha$ and SAPP $\beta$ using a duplex ELISA. We previously reported that APPV717I rostral neurons secrete a lower sAPP $\alpha$ : $\beta$ ratio than control rostral neurons, i.e., they favor $\beta$-secretase processing (Muratore et al., 2014a). We again observed this here, with a $\sim 30 \%$ decrease in sAPP $\alpha:$ SAPP $\beta$ with fAD mutation (Figure $3 \mathrm{~A}$ ). Compared with rostral neurons, neurons directed to caudal fates showed lower APPs $\alpha: \beta$ ratios in both the control (26\%) and fAD cultures (18\%) (Figure 3A), although this was only significantly lower in the control group, with a trend in the fAD group. Secretion of both SAPP $\alpha$ and SAPP $\beta$ was elevated in caudal neuronal cultures relative to rostral cultures (Figures 3B and 3C). This increase is more pronounced for SAPP $\beta$ (Figure 2C), resulting in a net decrease in the ratio of $\alpha$-to $\beta$-cleavage of APP. These results reveal that the relative contributions of $\alpha$ - and $\beta$-secretases to APP processing differ between neuronal fates.

Alpha secretases are members of the ADAM (a disintegrin and metalloprotease domain) family and $\beta$-secretases are encoded by two genes, $\beta$-site APP cleaving enzyme- 1 and -2 (BACE1 and BACE2). ADAMs and BACEs were expressed in both rostral and caudal cultures with similar levels expressed in each (Figure 3D). $\gamma$-Secretase is composed of PSEN, NCSTN, PEN2, and APH1 (Edbauer et al., 2003; Kimberly et al., 2003). There are two PSEN genes and two APH1 genes, and each $A P H 1$ gene has multiple isoforms. Multiple complexes of $\gamma$-secretase exist in human cells containing different PSEN and APH1 isoforms, and the makeup of the complexes can affect the type of A $\beta$ generated (Serneels et al., 2009). Expression levels of most of the $\gamma$-secretase components were unchanged between rostral and caudal cultures (Figure 3D). However, a subset of APH1 variants are differentially expressed between rostral and caudal fAD cultures (Figure 3D; Table S1), and this may contribute to the differences in $\mathrm{A} \beta$ generation observed.

The overall elevation in levels of APP cleavage products in caudal cultures points to a potential elevation in APP expression. To directly examine this, we measured expression of APP using a custom NanoString CodeSet (Figures 3E and 3F). Consistent with ELISA data of secreted APP metabolites, APP was significantly elevated in caudal neurons at the RNA level. Western blotting and quantification of lysates for APP holoprotein confirmed an increase in APP protein levels in neurons directed to caudal fates relative to rostral neurons (Figures $3 \mathrm{G}$ and $3 \mathrm{H}$ ).

Taken together, these results suggest that cells present in rostral and caudal cultures process APP differentially, with rostral cultures generating a higher level of pathogenic $A \beta 42$ relative to shorter $A \beta$ species. As outlined above and in other studies (Liao et al., 2016; Muratore et al., 2014a), these rostral cultures are composed of a mixture of astrocytes and different types of neurons. To examine a specific subset of rostral cells, we used the NGN2 direct induction protocol to generate relatively pure cultures of neurons most similar to excitatory projection neurons of the cerebral cortex (Zhang et al., 2013). Further, using CRISPRCas9 technology, we generated isogenic iPSC lines to fAD2B with correction of the APPV717I mutation, which will further be referred to here as "fAD ${ }^{\text {corr" }}$ (Figure $54 \mathrm{~A}$ ). We also isolated fAD clones that went through the CRISPR process, but did not have effective targeting of the mutant allele (referred to here as "fAD").

NGN2-induced neuron (iN) cultures from both $\mathrm{fAD}^{\text {corr }}$ and fAD express similar levels of APP, MAPT, MAP2, SYN1, and SYP (Figures S4B and S4C), with over 95\% of cells expressing NEUN and TAU following selection to eliminate

and teal bars, respectively. The " $\mathrm{n}$ " for each dataset is shown within the bar graph column. For each comparison, a one-way ANOVA with a Tukey's multiple comparisons post-test was performed, ${ }^{* *} p<0.01,{ }^{* *} p<0.001,{ }^{* * *} p<0.0001$. Data are represented as mean \pm SEM. (D-F) RNA was harvested from day 100 cultures and analyzed using a custom NanoString CodeSet. Data shown here are derived from three control lines and two fAD lines, over three differentiations. (D) The heatmap shows the mean expression levels of genes ( $n=6-10$ per condition) encoding components of the $\alpha-, \beta-$, and $\gamma$-secretases, for both rostral and caudal wild-type and fAD cultures. (E and F) APP expression data are shown between rostral and caudal cultures. Data in (E) are normalized to the average rostral data for each iPSC line. A Student's t test was performed, ${ }^{* *} p<0.01,{ }^{* * *} p<0.0001$. Data are represented as mean \pm SEM. Heatmap of expression of APP family members is shown, gene normalized (F). Raw NanoString data were normalized to housekeeping genes.

(G) A representative western blot (WB) from control day 100 total protein lysates.

(H) Quantification of WB data using densitometry from day 100 total protein lysates. For both rostral and caudal, $n=17$, derived from four differentiations. In $(\mathrm{H})$, a Student's $t$ test was performed, ${ }^{* *} \mathrm{p}<0.001$. Data are represented as mean \pm SEM.

See also Table S1. 
A
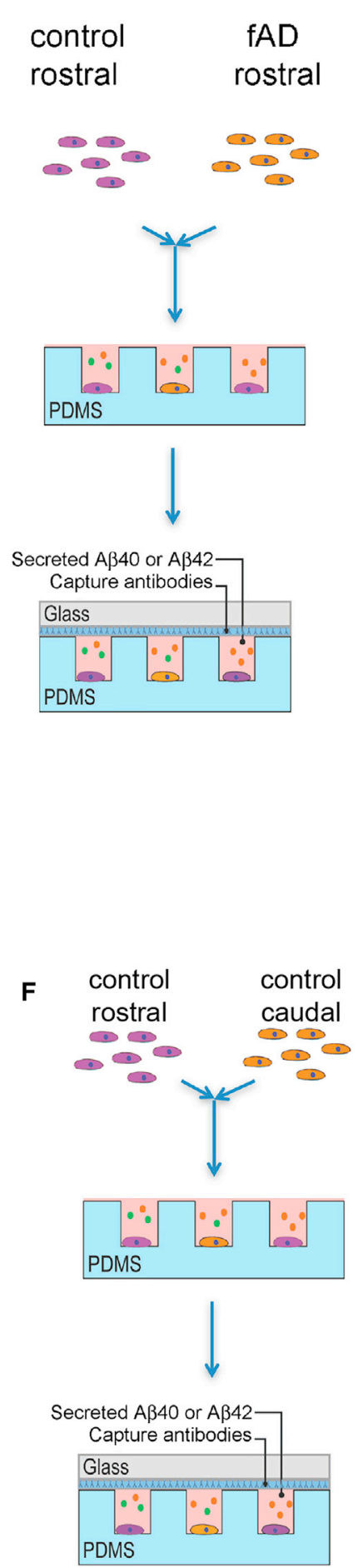

B
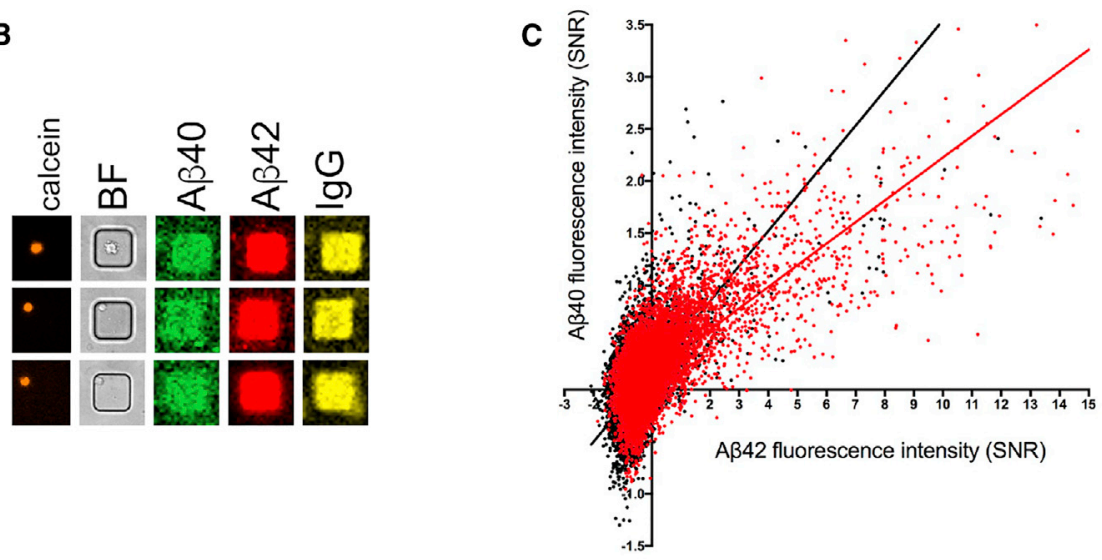

D
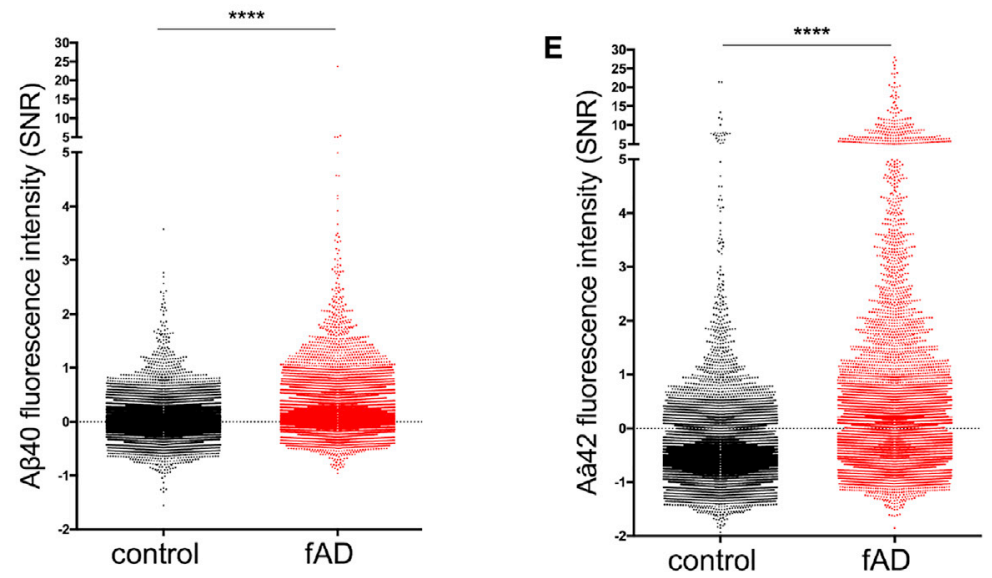

G
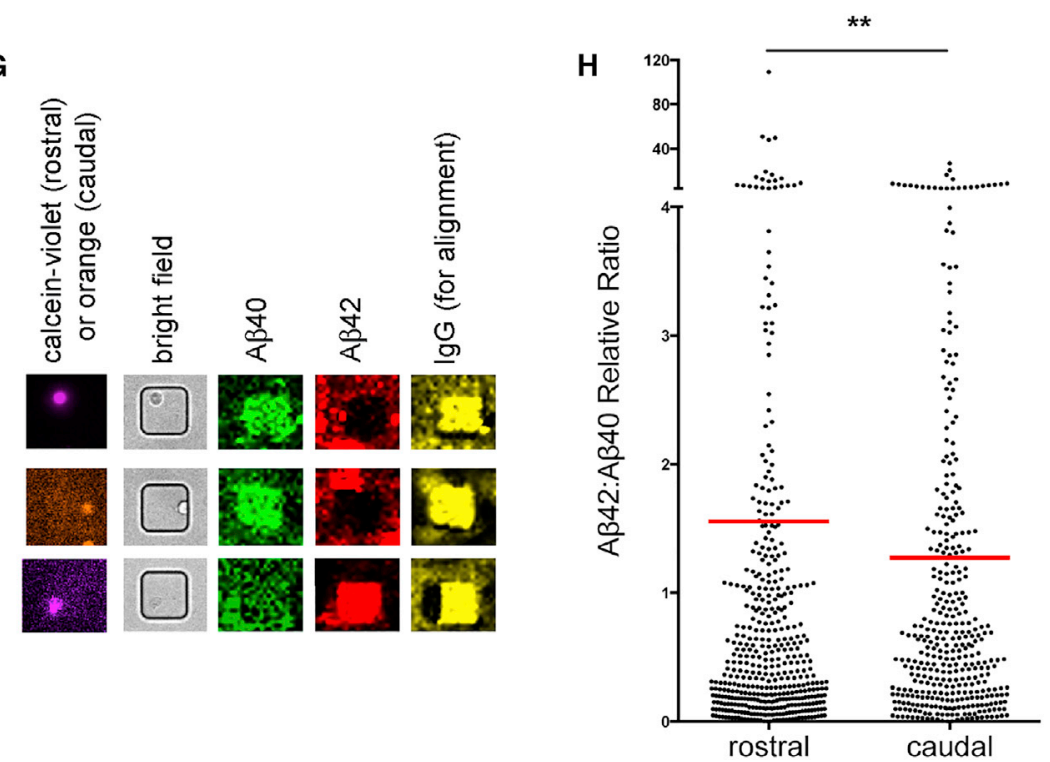

Figure 4. Single-Cell Analyses Support Differential A $\beta 40$ and A 342 Secretion by Distinct Neuronal Subtypes

(A) Control and FAD neurons were differentiated for $>100$ days and then dissociated, differentially labeled with either calcein-violet or calcein-orange, and plated into nanowell arrays at a density that favored a single cell per well. Cells in the nanowells were imaged, and then $A \beta 40$ or $A \beta 42$ were co-detected using microengraving. 
non-induced cells (data not shown). At day 28 of differentiation, we assayed 48-hr conditioned media using the triplex A $\beta$ ELISA, and fAD cultures displayed a significant increase in $\mathrm{A} \beta 42$ (Figures $2 \mathrm{E}$ and $\mathrm{S} 4 \mathrm{D}$ ), with no alterations in $A \beta 38$ and $A \beta 40$ (Figure S3D). This increase in $A \beta 42$ drives an elevation in $A \beta 42: 40$ ratio, in $f A D$ versus $f A D^{\text {corr }}$ neurons (Figure $2 \mathrm{~F}$ ), as well as a decreased $A \beta 38: 42$ ratio (Figure 2G). These results corroborate the effects of the fAD mutation on $\mathrm{A} \beta$ generation in rostral cultures, using an isogenic system and alternative differentiation protocol that yields a more pure culture of neuronal cells.

Single-Cell Analyses Support Differential A $\beta 40$ and A $\beta 42$ Secretion by Distinct Neuronal Subtypes Although cultures of iPSC-derived neurons with rostral versus caudal fates displayed differences in the cleavage patterns of APP, each of these culture types is heterogeneous in regard to the cell types present. "Rostral" cultures express markers that reflect a collection of upper and lower layer projection neurons, as well as excitatory and inhibitory neurons (Muratore et al., 2014a). "Caudal" cultures express markers that reflect neural subtypes found in the spinal cord and the hindbrain. We hypothesized that certain cell types within these cultures would contribute more to the observed effects on APP processing. We thus employed a single-cell approach to identify the effects of specific cell fates on APP cleavage within these cultures. We previously reported the development of a technology to examine $A \beta$ secretion at a single-cell level using microengraving (Liao et al., 2016). Here, we used this technique to identify the cells that secrete the highest levels of $A \beta 42$ and $\mathrm{A} \beta 40$.

Parallel rostral cultures of control and fAD neuronal fates were differentially labeled with calcein-violet and calceinorange, respectively, then mixed and plated in nanowells at a density that favored a single cell per well (Figure 4A).
These arrays were then imaged to acquire information about the number of living control and fAD cells present in each well. A glass slide coated with a capture antibody for $A \beta$ was placed over the nanowell array, sealing the wells off from their neighbors. Following an overnight incubation, slides were removed, and $A \beta 40$ and $A \beta 42$ were detected using differentially labeled antibodies (Figure 4B). Cells remaining in the nanowells were imaged for another live-cell marker, and analyzed. Figure 4C shows data from wells containing only a single living cell of control (black) or fAD (red) origin, with each dot representing data from a single cell. Using this method, the majority of cells of both control and fAD genotype do not secrete detectable $A \beta 40$ or $\mathrm{A} \beta 42$ (Figures $4 \mathrm{C}-4 \mathrm{E}$ ). Therefore, it is important to keep in mind that using this method we are only detecting $A \beta$ from the highest secretors. Despite this, similar to pooled culture data, we observe that fAD cells secrete higher levels of $A \beta$. Further, when $A \beta 40$ levels are graphed relative to $A \beta 42$ levels, the slope of the regression line is significantly shifted toward the A $\beta 42$ axis, reflecting an elevated 42:40 ratio (Figure $4 \mathrm{C}$ ). At the single-cell level, we find that this is driven by a higher number of fAD cells secreting extremely high levels of A $\beta 42$ relative to control cells (Figures $4 \mathrm{C}$ and $4 \mathrm{E}$ ).

While higher numbers of fAD cells secrete very high levels of AB42, smaller subsets of control cells secrete equally high levels as fAD cells. To probe which cells secrete the highest levels of $A \beta 40$ and $A \beta 42$ under non-fAD conditions, we differentially labeled rostral and caudal neural cells (Figure $4 \mathrm{~F}$ ), and measured $A \beta 40$ and $A \beta 42$ secretion by microengraving (Figure 4G). Interestingly, although these control rostral versus caudal cells do not show a significant change in the 42:40 ratio in pooled cultures (Figure 1C [YZ1/TZ1 lines]), at the single-cell level we detect a modest but significant decrease in the mean of the ratio of these highest $\mathrm{A} \beta$ secretors in caudal versus rostral cells (Figure $4 \mathrm{H}$ ).

(B) Example images of cells in nanowells that secrete high levels of $A \beta 40$ and $A \beta 42$.

(C) The signal-to-noise ratio (SNR = [foreground median - background median]/[STDEV background]) of fluorescence intensity for both $A \beta 40$ and $A \beta 42$ was measured for each well, and plotted. Each dot presents data from wells with single living cells, red dots/regression line from fAD ( $n=10,890$ cells), and black dots/regression line ( $n=25,603$ cells) from control. The difference in the slopes of the lines is significant, $\mathrm{p}<0.0001, \mathrm{DFn}=1, \mathrm{DFd}=25,603, \mathrm{~F}=571$.

( $D$ and $E$ ) Data in (C) re-graphed as scatterplots to compare $A \beta 40$ and $A \beta 42$ levels separately. While the majority of cells from both control and $F A D$ do not secrete detectable $A \beta 40$ or $A \beta 42$, the mean secretion of both is elevated in $F A D$ relative to control. Mann-Whitney test performed, ${ }^{* * * *} p<0.001$.

(F) Control rostral and caudal neurons were differentiated for $>100$ days and then dissociated, differentially labeled with either calceinviolet or calcein-orange, and plated into nanowell arrays at a density that favored a single cell per well. Cells in the nanowells were imaged, and then $A \beta 40$ or $A \beta 42$ were co-detected using microengraving.

(G) Example images of cells in nanowells that secrete high levels of $A \beta 40$ or $A \beta 42$.

(H) SNR of fluorescence intensity for both A 440 and A 42 was measured for each well, and a relative ratio of $42: 40$ calculated. Shown is a scatterplot of data from wells with single living cells, where each data point reflects data from a single cell. Only wells that had single live cells and detectable $A \beta 40$ and $A \beta 42$ are shown. Red lines show means of the ratio for the rostral and caudal populations. Mann-Whitney test performed, ${ }^{* *} p<0.005$.

See also Figure S5 and Tables S2 and S3. 

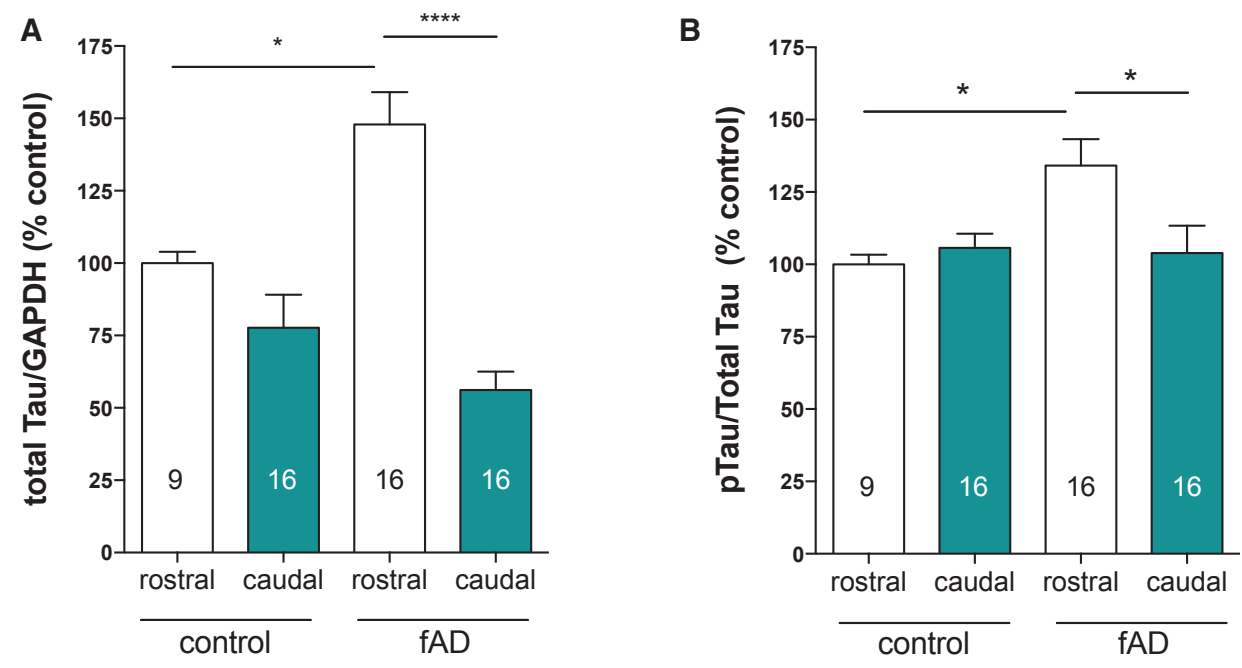

C

Ngn2-iN-rostral

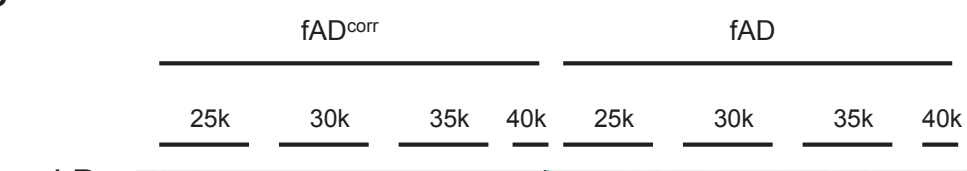

$\mathrm{kDa}$

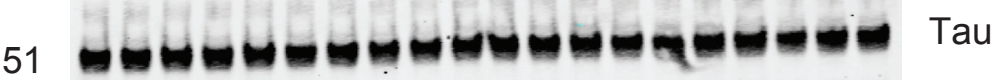

51

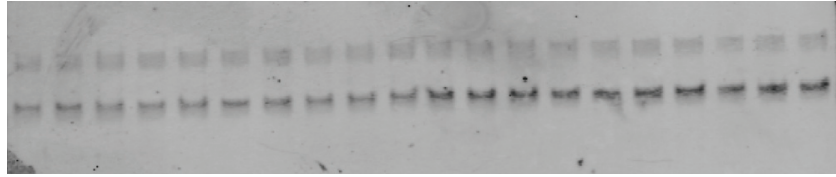

39

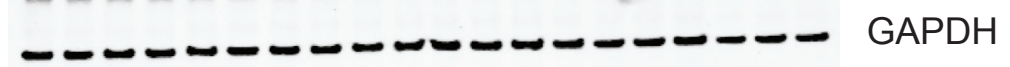

D

E
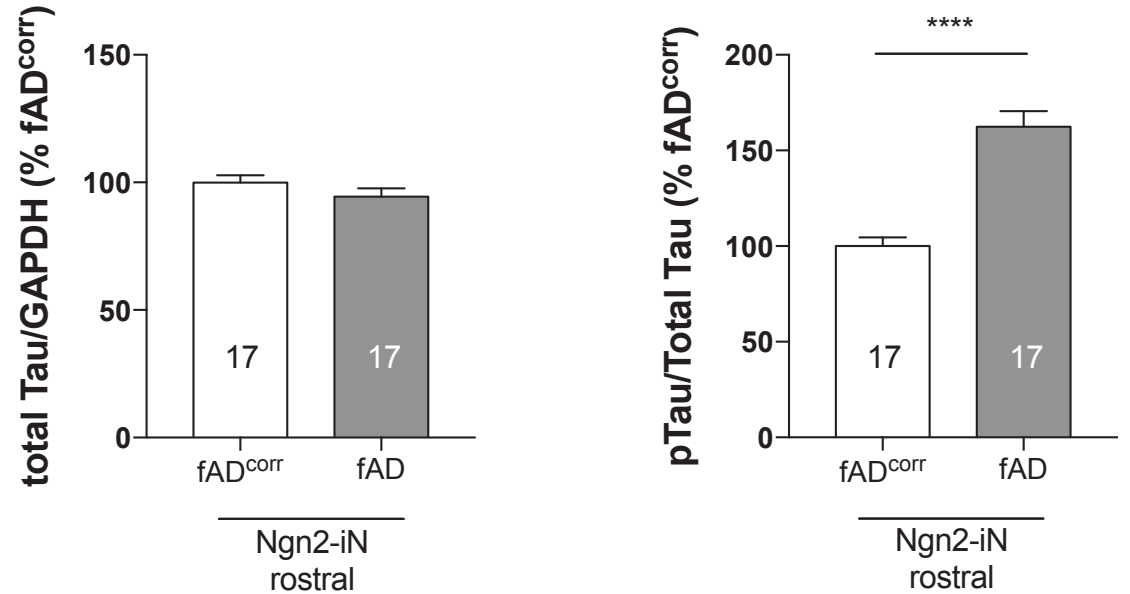

Ngn2-iN

rostral 
To profile cell-type marker expression in sets of the highest $A \beta$ secretors, individual cells were retrieved from the nanowells following single-cell detection of $A \beta$, and their mRNAs profiled using our custom NanoString CodeSet. Figure S5A and Table S2 show data from wells containing only a single living cell. The majority of $A \beta$-secreting cells expressed the general neuronal markers DLG4,SYN1, and MAPT (Figure S5A; Table S2). The cells that secreted the highest levels of $A \beta 42$ preferentially expressed markers of the cortex/forebrain (CUX1, SATB1, FEZF2, and TBR1) (Figure S5A; Table S2), and did not express markers of the cerebellar/spinal cord region (HOXB13, HOXB4, ISL-1) (Figure S5A; Table S2).

In this analysis, high $A \beta 40$ cells appear to preferentially express inhibitory (SLC32A1, GAD1, NKX2-1, and CALB2) versus excitatory markers (SLC17A7, GRIN1, and GRIK1), and high $\mathrm{A} \beta 42$ secretors preferentially expressed excitatory markers versus inhibitory markers (Figure S5A; Table S2). Interestingly, when we examined NanoString data from pooled rostral and caudal cultures, we found that caudal neurons had a more "inhibitory" profile, with higher mRNA expression of GAD1 and SLC32A1/VGAT, and lower mRNA expression of SLC17A7/VGLUT1 and SLC17A6/ VGLUT2, compared with rostral neurons (Figure S5B). Publically available RNA sequencing data from postmortem human tissue (Mayo Brain Gene Expression, led by Dr. Nilufer Taner and Dr. Steven G. Younkin) revealed similar differential expression of these genes between cerebellum and temporal cortex samples (Figure S5C). These data raise the possibility that the inhibitory/excitatory balance of neuronal activity may contribute to the differential effects on $A \beta$ observed here. We therefore examined spontaneous neuronal activity of control and fAD cultures directed to rostral and caudal fates using multi-electrode arrays (MEAs). Analyses of baseline (non-stimulated) activity suggest that rostral and caudal cultures display similar levels of spontaneous firing and bursting, with no significant differences in waveform shape parameters (Table S3). These results suggest that spontaneous activity is not dramatically different between rostral and caudal cultures. However, a deeper and more-refined analyses of activity in these cultures would be necessary to rule out a contribution of neuronal activity to our findings.

Rostral versus Caudal Neurons Exhibit Differential TAU Responses to the fAD Mutation and to Soluble Brain Extracts from LOAD Subjects

The results described above provide evidence that APP processing differs as a function of regional neuronal identity. We next interrogated whether this altered processing has a functional consequence for an AD-relevant downstream phenotype. We previously reported that neurons expressing the APPV717I mutation, directed to forebrain cortical fates, exhibit higher levels of total TAU and phospho-TAU (Muratore et al., 2014a), and we again observed this effect (Figures 5A and 5B, white bars). Importantly, the increases in total TAU and phospho-TAU levels caused by the fAD mutation in rostral, forebrain neurons were not observed in parallel cultures directed to caudal fates (Figures $5 \mathrm{~A}$ and $5 \mathrm{~B}$, teal bars). When phospho-TAU levels were normalized to total TAU, there was a $\sim 20 \%$ decrease in phosphoTAU in fAD caudal neurons compared with fAD rostral neurons, achieving a ratio similar to neurons lacking the fAD mutation (Figures 5B and S6). Similarly, in the lentiviral overexpression model system in which control rostral and caudal cultures were transduced with the APPV717I virus (Figure S3), we observe an increase in phospho-TAU levels with expression of fAD mutation in rostral, forebrain neurons. This effect was not observed in parallel cultures directed to caudal fates, despite clear overexpression of APPV717I (Figure S3F). As we have previously shown, the fAD-dependent increase in phospho-TAU is seen at latestage differentiation time points (day 80+). In the lentiviral overexpression model system, we indeed observe an increase in phospho-TAU in APPV717I cultures at day 100, but this phenotype is not present at an earlier time point (day 40; Figure S3E).

To examine effects of APPV717I on TAU in an isogenic model, we examined phospho-TAU and total TAU in the NGN2-iNs derived from $\mathrm{fAD}$ and $\mathrm{fAD}^{\mathrm{corr}}$ lines. In this system, fAD neurons again exhibited an elevated phosphoTAU/total TAU ratio relative to control (fAD ${ }^{\text {corr }}$ ) cultures

Figure 5. Rostral versus Caudal Cultures Respond Differentially to the fAD Environment

(A and B) Control and FAD rostral and caudal cultures at 100 days of differentiation were lysed, and total TAU (A) and phospho-TAU (B) levels were measured by WB and densitometry. The " $n$ " for each dataset is shown within the bar graph column. There are four lines represented, over four independent rounds of differentiation. One-way ANOVA with a Tukey's multiple comparisons test performed, * $p<0.05$, $* * * * p<0.0001$. Data are represented as mean \pm SEM.

(C-E) Data are from day $28 \mathrm{Ngn} 2-\mathrm{iN}$ cultures. (C) A representative WB from total protein lysates. Various cell densities of $25 \mathrm{k}(\mathrm{n}=3), 30 \mathrm{k}$ $(n=3), 35 k(n=3)$, and 40k $(n=1)$ were plated and analyzed, and protein normalized prior to gel loading. (D and E) Quantification of WB data using densitometry from total protein lysates. Total TAU (D) and phospho-TAU (E) levels were measured by WB and densitometry. The " $\mathrm{n}$ " for each dataset is shown within the bar graph column. There are three independent rounds of differentiation represented. For each comparison, a Student's t test was performed, ${ }^{* * *} p<0.0001$. Data are represented as mean \pm SEM.

See also Figure 56 . 
(Figures 5C-5E). Figure 5C shows a representative western blot of total protein lysates. Total TAU and phospho-TAU levels are quantified in Figures 5D and 5E. Interestingly, while this TAU phenotype is observed in these iNs, the total TAU/GAPDH elevation observed in rostral EB-derived cultures is not observed (Figure 5D). Taken together, these results support an effect of the APPV717I fAD mutation on TAU proteostasis in multiple forebrain cellular models.

The experiments described above suggest that neuronal subtypes can respond differentially to an fAD mutation, i.e., they can show differences in both APP processing and TAU homeostasis. While the type and level of $A \beta$ generated in rostral versus caudal neurons is different, it is not clear whether control neurons would respond differentially to the same AD-relevant pathogenic stimulus. To test this, we exposed control (non-fAD containing) neurons differentiated in parallel to rostral and caudal fates to a Tris-buffered saline (TBS)-soluble extract from LOAD postmortem brain (AD-TBS). Similarly prepared AD-TBS extracts have been shown to inhibit long-term potentiation and elevate phospho-TAU when added to slice cultures, and to affect memory in rodents in an $A \beta$-dependent manner (Jin and Selkoe, 2015; Jin et al., 2011; Shankar et al., 2008).

AD-TBS treatment of rostral or caudal neurons for 2 days had no qualitative effect on the morphology of neurons (Figure 6A). However, quantification by both ELISA (Figure 6B) and western blot (Figure 6C) revealed that ADTBS treatment induced an increase in phosphorylation of TAU relative to overall TAU levels in rostrally directed neurons, but the same extract had no effect on phospho-TAU levels in caudal neurons differentiated and treated in parallel. No differences in total TAU were observed with AD-TBS treatment (Figure 6D). Treatment with AD-TBS extract from human brain introduces a relatively high level of $\mathrm{A} \beta$ to the cells: total $\mathrm{A} \beta$ levels in these AD-TBS extracts (19 $\mathrm{ng} / \mathrm{mL}$ ) are $~ 50$-fold higher than the levels endogenously produced by the iPSC-derived neuronal cultures $(0.4 \mathrm{ng} / \mathrm{mL})$. The effect of AD-TBS extract on phosphoTAU in the rostral neurons was significantly decreased by co-administering a polyclonal antibody (AW7) to $A \beta$ (Figures $6 \mathrm{~B}$ and $6 \mathrm{C}$ ), indicating that the soluble $\mathrm{A} \beta$ assemblies present in AD-TBS extract mediate the induced elevation in TAU phosphorylation.

\section{DISCUSSION}

We reported previously that an fAD APP mutation elevates the A $\beta 42: 40$ ratio and the total TAU and phospho-TAU levels in rostral, forebrain neuronal cultures (Muratore et al., 2014a). The elevated levels of TAU observed in these neurons were rescued by co-treatment with an A $\beta$-neutralizing antibody, suggesting that $A \beta$ altered total TAU and phospho-TAU levels in this system (Muratore et al., 2014a). Here, we show that caudally directed fAD neurons secrete $A \beta$ with a reduced $A \beta 42: 40$ ratio relative to rostral neurons, and that fAD-induced elevations in total TAU and phospho-TAU are absent when cells are directed to caudal neuronal fates. While APP processing differs significantly in multiple ways between rostral and caudal fates, this alone may not be sufficient to drive the differential fAD TAU phenotype observed. To interrogate the potential contribution of responsiveness to neurotoxic species in the absence of an fAD mutation, we exposed control rostral and caudal neurons to the same soluble species isolated directly from LOAD brains. We found that AD brain extract induces an elevation of phospho-TAU levels in rostral, forebrain neurons in an $\mathrm{A} \beta$-dependent manner, but that caudal neurons treated in parallel did not show this response. Taken together, our data reveal that neurons directed to rostral versus caudal fates have intrinsic differences in both their generation of and responses to $\mathrm{AD}$-relevant $\mathrm{A} \beta$ species.

In our model system, we direct cells to what we refer to as rostral and caudal fates. The protocols that we use are well established and described in prior studies (Hu and Zhang, 2009; Lee et al., 2007; Li et al., 2008; Muratore et al., 2014b, 2014a; Zeng et al., 2010). The differentiations to rostral and caudal fates are performed in parallel, with the only difference between culture conditions being the addition of Shh and RA during a critical developmental window. In rostral cultures, we generate a heterogeneous mixture of upper and lower layer cortical neurons of both inhibitory and excitatory fates. Caudal cultures also are heterogeneous mixtures of multiple neuronal fates, but express classical markers of hindbrain and spinal cord, such as $H O X$ genes, $I S L-1$, and $H B 9$, and do not express markers of cortical fates. The levels of general neuronal markers at the RNA and protein levels are not significantly different between these populations, and the cells appear morphologically indistinguishable. At late stages of differentiation (>day 40), astrocytes are present in both rostral and caudal cultures, but the relative numbers of cells expressing markers of neurons and astrocytes is not significantly different between rostral and caudal cultures. APP, and genes encoding $\alpha-, \beta$-, and $\gamma$-secretase, are expressed in both culture types. Notwithstanding these similarities, we observed multiple differences in APP metabolism and cellular responses to $A \beta$. What is different about these distinct neural subtypes that leads to altered generation of and response to $A \beta$ ?

One possibility is that differences in electrophysiological activity between these culture types may contribute to observed differences in levels of $\mathrm{A} \beta$ generation. In studies 
A
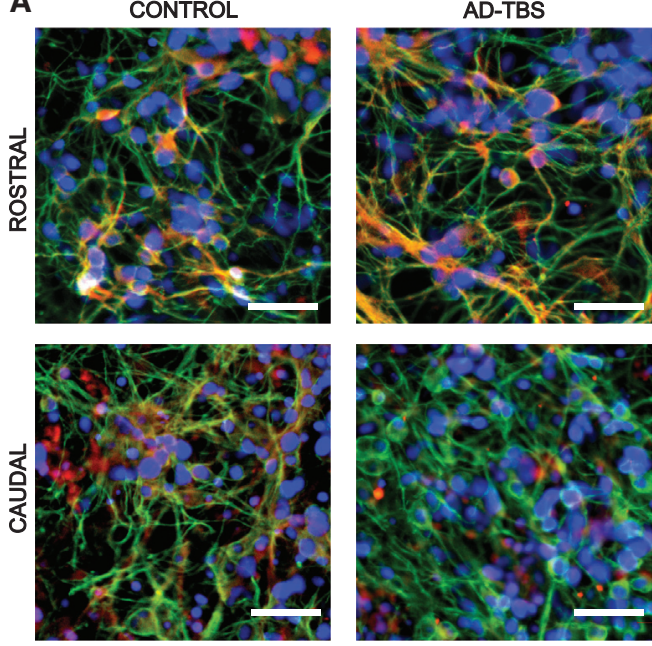

C

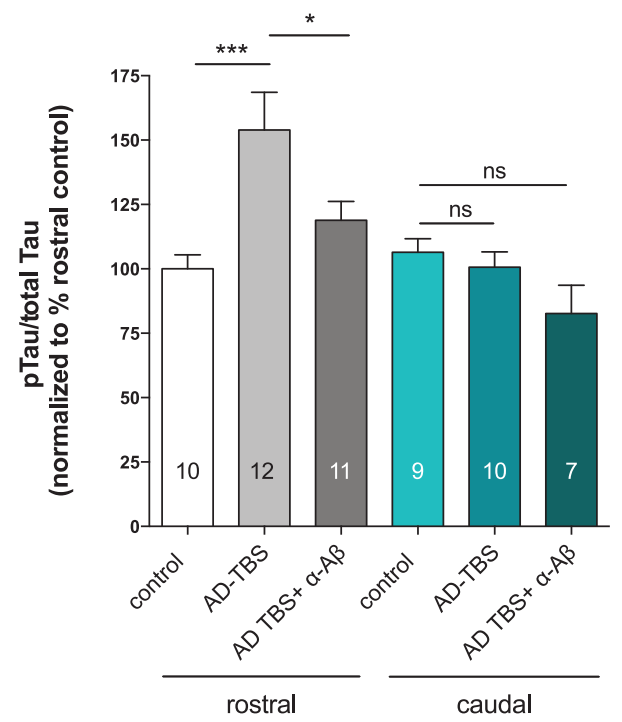

$\mathrm{AD}-\mathrm{TBS}+\alpha-\mathrm{A} \beta$

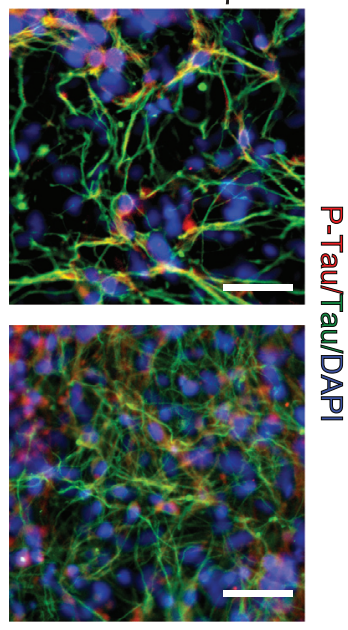

D
B

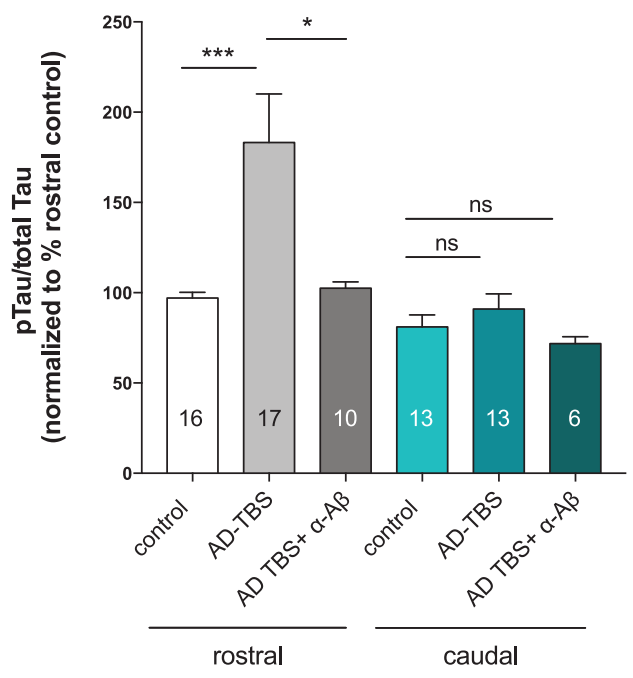

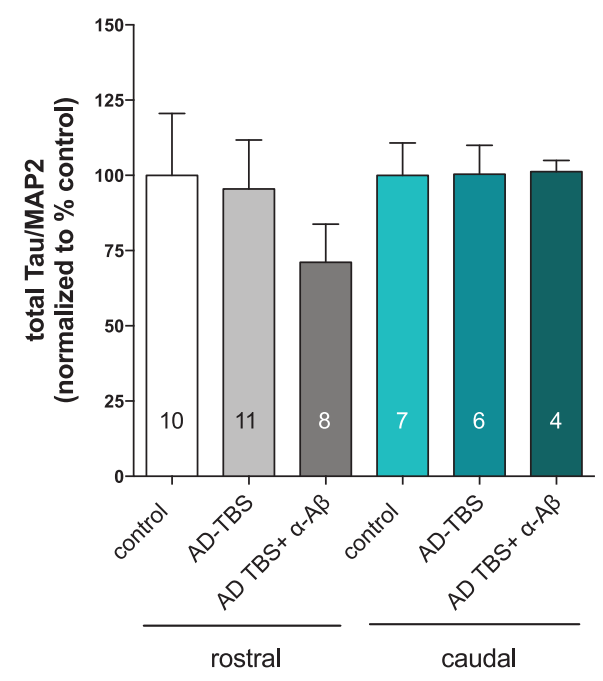

Figure 6. Rostral versus Caudal Neural Cultures Respond Differentially to the LOAD Environment

At 80-100 days of differentiation, control iPSC-derived rostral and caudal neurons were treated for 2 days with TBS-soluble extract of sporadic $A D$ postmortem brain, with and without co-administration of a polyclonal $A \beta$-neutralizing antibody (AW7). Cultures were fixed and immunostained for total and phospho-TAU (A). Images presented here are only for visualization of the cells; relative intensities are not acquired in a manner that allows for detection of quantitative differences. Scale bars, $100 \mu \mathrm{m}$. Parallel rostral and caudal cultures were lysed and subjected to ELISA (B) and WB (C and D). Lysates were run on a phospho (Thr 231)/total TAU MSD ELISA and phospho-TAU normalized to total TAU (B). For WBs, quantification by densitometry was performed and phospho-TAU was normalized to total TAU (C) or else total TAU was normalized to MAP2 (D). The " $\mathrm{n}$ " for each dataset is shown within the bar graph column. For each comparison, a one-way ANOVA with a Tukey's multiple comparisons post-test was performed, ${ }^{*} p<0.05,{ }^{* *} p<0.001$. ns, not significant. Data are represented as mean \pm SEM, over four independent rounds of differentiation.

using in vivo microdialysis in mutant human APP transgenic mice, different regions of the brain showed different levels of soluble human $A \beta$, and higher levels of $A \beta$ were correlated with elevated neuronal activity (Bero et al., 2011). Using MEAs, we find that both rostral and caudal neurons show similar levels of spontaneous activity (Table S3). However, the precise excitatory/inhibitory balance of activity may be different between culture types, and future studies are warranted to more deeply interrogate this question. 
In addition to an effect on overall $\mathrm{A} \beta$ production, our data suggest that neurons of a rostral fate, which are more affected by $A D$, secrete $A \beta$ that has a higher level of the pathogenically critical $A \beta 42: 40$ ratio than neurons with more caudal fates of the hindbrain and spinal cord. While this change in ratio is modest, it is highly reproducible across multiple differentiations and lines, and mirrors the ratio change observed in $\mathrm{fAD}$ brain. Importantly, it has been demonstrated that it is the types of $A \beta$ generated, rather than overall $A \beta$ levels, which determine the degree of neurotoxicity of $A \beta$. Strong evidence for this concept emerges from the genetics of fAD. Numerous mutations in APP and PSEN elevate the A $\beta 42: 40$ ratio, many by increasing $A \beta 42$, but some through decreasing $A \beta 40$ (Bentahir et al., 2006; Scheuner et al., 1996). A recent study investigating $A \beta$ levels in postmortem brains of a large number of PSEN mutation carriers showed that impaired carboxypeptidase-like activity was a consistent feature across mutations (Szaruga et al., 2015), a feature that we also observe with an APP fAD mutation. Further, elevated A $\beta 40$ levels have been shown to actually be anti-amyloidogenic; that is, they do not lead by themselves to $A \beta$ deposition and can mitigate the effects of elevated $A \beta 42$ levels in APP transgenic mice (Kim et al., 2007; McGowan et al., 2005). Moreover, in biochemical studies, it has been shown that even a subtle alteration in the $A \beta 42: 40$ ratio (similar to that which we observe here) can dramatically affect $A \beta$ aggregation kinetics as well as the cytotoxicity of $A \beta$ preparations (Kuperstein et al., 2010). Thus, we hypothesize that the lower $A \beta 42: 40$ and elevated A $\beta 38: 42$ ratio of caudal cultures contributes to a protective effect on the downstream alterations in total TAU and phospho-TAU.

The length of $A \beta$ is determined by the site within APP of the initial endopeptidase cleavage by $\gamma$-secretase as well as the efficiency of subsequent carboxypeptidase-like processing. Multiple variables have been shown to affect these activities, including local lipid composition and the composition of $\gamma$-secretase itself. For example, increased fatty acid chain length decreases the A $\beta 42: 40$ ratio, while gangliosides increase this ratio (Holmes et al., 2012). These differences have direct effects on membrane thickness and fluidity, which in turn may alter $\gamma$-cleavage of APP. In fact, there is evidence that $A \beta 42$ and $A \beta 40$ are preferentially generated in the endoplasmic reticulum and trans-Golgi network, respectively, perhaps due to different membrane thicknesses (Hartmann et al., 1997). In addition to membrane composition, the presence of PSEN1 versus PSEN2 and/or different APH1 proteins also can affect the type of A $\beta$ generated (Acx et al., 2014; Serneels et al., 2009). There is evidence for regional differences in expression of APH1 variants, with knock out of $\mathrm{APH} 1 \mathrm{~B}$ and $\mathrm{C}$ in mice resulting in greater effects on $\gamma$-secretase activity in the cerebellum and brain stem than in the cerebral cortex (Serneels et al., 2005).

In our bulk cultures, it is not clear whether differences in APP processing were intrinsically programmed or if APP processing was affected by neighboring cells. However, using our single-cell microengraving approach, we could isolate individual cells from their neighbors and still observe significant effects on $A \beta 42$ and $A \beta 40$ secretion in more caudal versus more rostral neurons. This result suggests that effects on $\mathrm{A} \beta 42$ and $\mathrm{A} \beta 40$ are in part intrinsically encoded in a cell-autonomous manner.

Using a controlled, reductionist approach, we find that neuronal cell fate affects the generation of, and responses to $\mathrm{A} \beta$, but how does this relate to the $\mathrm{AD}$ brain? In sporadic $\mathrm{AD}$ and many cases of $\mathrm{FAD}$, the hindbrain and spinal cord are relatively spared of $\mathrm{A} \beta$ and TAU pathology (and resultant symptoms), while the limbic and cerebral cortices are highly vulnerable to accumulation of plaques and tangles, synapse loss, and neuronal death. Multiple mechanisms are likely to contribute to this differential vulnerability, including important $A \beta$ and TAU clearance mechanisms, which were not addressed here. Obvious differences between our in vitro culture system and patient brains preclude a direct comparison of $A \beta$ generation and secretion in different brain regions. Nonetheless, there is published evidence of differential levels of $A \beta 40$ and $\mathrm{A} \beta 42$ across rostral and caudal regions in the AD brain. In an in-depth study of $A \beta$ species in $\mathrm{AAD}$ and $\mathrm{LOAD}$ postmortem brain, regional differences in $A \beta 40$ and $A \beta 42$ accumulation in soluble and insoluble brain fractions were found: fAD brain had a disproportionate accumulation of $\mathrm{A} \beta 40$ compared with LOAD brain in the cerebellum, while A $\beta 42$ levels were generally much lower in cerebellum than cerebral cortex for both fAD and LOAD (Shinohara et al., 2014). Similarly, in another study using immunoprecipitation and mass spectrometry, cerebellum contained much higher levels of $A \beta 40$ compared with $A \beta 42$, while cortex contained relatively higher levels of $A \beta 42$, in both LOAD and fAD brain samples (Portelius et al., 2010). While the human brain is clearly much more complex than our cell culture models, the integration of our findings in vitro with further studies of human brain may aid in our understanding of why some brain regions are strongly affected in neurodegenerative diseases, whereas others are relatively resistant.

\section{EXPERIMENTAL PROCEDURES}

\section{iPSC Culture Conditions}

All human iPSC work was approved by the BWH IRB (protocol 2015P001676/BWH and 2015P002521/BWH). iPSCs were cultured in iPSC medium with FGF2 (PeproTech). iPSCs were maintained on a mouse embryonic fibroblast feeder layer (GlobalStem). Cells were 
split as necessary based on colony growth (5-6 days). Differentiating colonies were removed from the plate prior to splitting. For full media recipes see Supplemental Experimental Procedures.

\section{iPSC Differentiation}

For the induction of neural cultures (rostral and caudal), iPSCs were differentiated using an EB-based protocol (Zeng et al., 2010) that was further optimized (Muratore et al., 2014b). For the Neurogenin 2-iN (Ngn2-iN) differentiation, iPSCs were differentiated following the protocol in (Zhang et al., 2013) with minor modifications. Cultures were treated with doxycycline $(2 \mu \mathrm{g} / \mathrm{mL})$ on day 1 to induce differentiation, fed with a series of medium changes, and harvested at day 21 (see Supplemental Experimental Procedures).

\section{RNA Analyses}

\section{RNA Sequencing}

Total RNA from rostral and caudal cultures were assayed for quality on an Agilent TapeStation 4200. Double-stranded cDNA was synthesized using the SuperScript III Reverse Transcriptase protocol using random hexamers on the polyA-RNA eluate. Libraries were generated by processing the doublestranded cDNA product through the Illumina Nextera tagmentation library protocol. Multiplexed libraries were sequenced on an Illumina NextSeq 500 to a depth of 433 million paired-end reads (75 bases per read) total. Hox gene-level expression data were extracted from the expression table and the mean plotted as a heatmap.

qPCR

qPCR was performed using Fast SYBR Green Master Mix and run on a ViiA 7 System (Applied Biosystems). RNA was purified from individual samples and processed through a PureLink RNA Mini Kit (Ambion), followed by reverse transcription using SuperScript II (Invitrogen).

NanoString Assay

We utilized a custom probe set designed by NanoString Technologies to analyze gene expression for 150 genes from each sample. Assays were performed using the NanoString protocols per the manufacturer's instructions.

\section{A $\beta$, sAPP $\alpha /$ sAPP $\beta$, and Phospho-TAU/Total TAU ELISA Assays}

ELISA assays were carried out using the reagents, protocols, and imager manufactured by Meso Scale Diagnostics. Media or lysates (as indicated) were collected after $48 \mathrm{hr}$ from day 40 to 50 cultures (media) or day 80 to 100 cultures (lysates), and analyzed using the 6E10 Abeta Triplex, sAPP $\alpha / \mathrm{sAPP} \beta$, or phospho (Thr 231)/total TAU ELISA assays. Data were normalized to total RNA or total protein where indicated.

\section{AD-TBS Treatments}

For AD-TBS treatments of iPSC-derived cultures, day 80 neural cultures were treated for 2 days with TBS-soluble extract of LOAD postmortem brain $(1: 20$ of $19 \mathrm{ng} / \mathrm{mL}$ total $\mathrm{A} \beta)$, as calculated by the V-PLEX A $\beta$ Peptide Panel 1 (6E10) Kit (Meso Scale Diagnostics), with and without co-administration of a polyclonal $A \beta$-neutraliz- ing antibody (AW7) (McDonald et al., 2012). The rabbit polyclonal antibody AW7 was used at $30 \mu \mathrm{g} / \mathrm{mL}$ for antibody treatment experiments.

\section{Microengraving and Printing}

Aged-differentiated (>100 days) control rostral and caudal neurons were dissociated and stained with live markers. Cells were loaded from suspension $\left(1-2 \times 10^{5}\right.$ cells $\left./ \mathrm{mL}\right)$ onto PDMS arrays, which favors $0-2$ cells per well. Single-cell detection of $\mathrm{A} \beta$ was performed using icroengraving as described (Liao et al., 2016). See Supplemental Experimental Procedures for more details.

\section{Statistical Analysis}

Data were analyzed using GraphPad Prism 6 software. Values are expressed as mean \pm SD or \pm SEM, as indicated by figure legend text. Statistical significance was tested by either an unpaired Student's t test (two-tailed) or by one-way ANOVA with a Tukey's multiple comparisons post-test, as indicated. Statistically significant differences were determined by $\mathrm{p}$ values less than 0.05 . All replicates, experimental "n," iPSC lines and differentiations used are noted in the figure legends.

\section{SUPPLEMENTAL INFORMATION}

Supplemental Information includes Supplemental Experimental Procedures, six figures, and three tables and can be found with this article online at https://doi.org/10.1016/j.stemcr.2017.10. 015 .

\section{AUTHOR CONTRIBUTIONS}

Conceptualization, C.R.M. and T.L.Y.-P.; Methodology, C.R.M., M.L., P.S., D.G.C., J.C.L., and T.L.Y.-P.; Software, J.M.N.; Formal Analysis, C.R.M., R.V.P., M.L., J.M.N., and T.L.Y.-P.; Investigation, C.R.M., C.Z., M.L., M.A.F., W.M.T., V.N.L., R.V.P., H.C.R., A.H., D.G.C., and T.S.; Resources, M.Z., D.A.B., S.N., J.C.L., D.J.S., and T.L.Y.-P.; Writing - Original Draft, C.R.M., C.Z., D.J.S., and T.L.Y.-P.; Writing - Review \& Editing, C.R.M., H.C.R., R.V.P., D.J.S., and T.L.Y.-P.; Visualization, C.R.M. and T.L.Y.-P.; Supervision, C.R.M. and T.L.Y.-P.; Funding Acquisition, T.L.Y.-P.

\section{ACKNOWLEDGMENTS}

We thank Hyo Lee and Julie Merchant for technical assistance, Ting Yang for providing the AD postmortem brain extract, and Dominic Walsh for providing the AW7 antibodies. We thank Dominic Walsh and Matthew LaVoie for helpful discussions and critical reading of portions of the manuscript. This work is part of the AD Deep Phenotyping program supported by a gift from Rick and Nancy Moskovitz and is supported by the BrightFocus Foundation, the Brigham Research Institute, NIH AG056011, and NIH AG049864 (to T.L.Y.-P.) and T32AG000222.

Received: May 2, 2017

Revised: October 17, 2017

Accepted: October 18, 2017

Published: November 16, 2017 


\section{REFERENCES}

Acx, H., Chávez-Gutiérrez, L., Serneels, L., Lismont, S., Benurwar, M., Elad, N., and De Strooper, B. (2014). Signature amyloid $\beta$ profiles are produced by different $\gamma$-secretase complexes. J. Biol. Chem. 289, 4346-4355.

Bentahir, M., Nyabi, O., Verhamme, J., Tolia, A., Horré, K., Wiltfang, J., Esselmann, H., and De Strooper, B. (2006). Presenilin clinical mutations can affect gamma-secretase activity by different mechanisms. J. Neurochem. 96, 732-742.

Bero, A.W., Yan, P., Roh, J.H., Cirrito, J.R., Stewart, F.R., Raichle, M.E., Lee, J.-M., and Holtzman, D.M. (2011). Neuronal activity regulates the regional vulnerability to amyloid- $\beta$ deposition. Nat. Neurosci. 14, 750-756.

Bertram, L., Lill, C.M., and Tanzi, R.E. (2010). The genetics of Alzheimer disease: back to the future. Neuron 68, 270-281.

Edbauer, D., Winkler, E., Regula, J.T., Pesold, B., Steiner, H., and Haass, C. (2003). Reconstitution of gamma-secretase activity. Nat. Cell Biol. 5, 486-488.

Hartmann, T., Bieger, S.C., Brühl, B., Tienari, P.J., Ida, N., Allsop, D., Roberts, G.W., Masters, C.L., Dotti, C.G., Unsicker, K., et al. (1997). Distinct sites of intracellular production for Alzheimer's disease A beta40/42 amyloid peptides. Nat. Med. 3, 1016-1020.

Holmes, O., Paturi, S., Ye, W., Wolfe, M.S., and Selkoe, D.J. (2012). Effects of membrane lipids on the activity and processivity of purified $\gamma$-secretase. Biochemistry 51, 3565-3575.

Hu, B.-Y., and Zhang, S.-C. (2009). Differentiation of spinal motor neurons from pluripotent human stem cells. Nat. Protoc. 4, 12951304.

Israel, M.A., Yuan, S.H., Bardy, C., Reyna, S.M., Mu, Y., Herrera, C., Hefferan, M.P., Van Gorp, S., Nazor, K.L., Boscolo, F.S., et al. (2012). Probing sporadic and familial Alzheimer's disease using induced pluripotent stem cells. Nature 482, 216-220.

Jin, M., and Selkoe, D.J. (2015). Systematic analysis of time-dependent neural effects of soluble amyloid $\beta$ oligomers in culture and in vivo: prevention by scyllo-inositol. Neurobiol. Dis. 82, 152-163. Jin, M., Shepardson, N., Yang, T., Chen, G., Walsh, D., and Selkoe, D.J. (2011). Soluble amyloid beta-protein dimers isolated from Alzheimer cortex directly induce Tau hyperphosphorylation and neuritic degeneration. Proc. Natl. Acad. Sci. USA 108, 5819-5824.

Kim, J., Onstead, L., Randle, S., Price, R., Smithson, L., Zwizinski, C., Dickson, D.W., Golde, T., and McGowan, E. (2007). Abeta40 inhibits amyloid deposition in vivo. J. Neurosci. 27, 627-633.

Kimberly, W.T., LaVoie, M.J., Ostaszewski, B.L., Ye, W., Wolfe, M.S., and Selkoe, D.J. (2003). $\gamma$-Secretase is a membrane protein complex comprised of presenilin, nicastrin, aph-1, and pen-2. Proc. Natl. Acad. Sci. USA 100, 6382-6387.

Koch, P., Tamboli, I.Y., Mertens, J., Wunderlich, P., Ladewig, J., Stüber, K., Esselmann, H., Wiltfang, J., Brüstle, O., and Walter, J. (2012). Presenilin-1 L166P mutant human pluripotent stem cellderived neurons exhibit partial loss of $\gamma$-secretase activity in endogenous amyloid- $\beta$ generation. Am. J. Pathol. 180, 2404-2416. Kondo, T., Asai, M., Tsukita, K., Kutoku, Y., Ohsawa, Y., Sunada, Y., Imamura, K., Egawa, N., Yahata, N., Okita, K., et al. (2013). Modeling Alzheimer's disease with iPSCs reveals stress phenotypes associated with intracellular $A \beta$ and differential drug responsiveness. Cell Stem Cell 12, 487-496.

Kuperstein, I., Broersen, K., Benilova, I., Rozenski, J., Jonckheere, W., Debulpaep, M., Vandersteen, A., Segers-Nolten, I., Van Der Werf, K., Subramaniam, V., et al. (2010). Neurotoxicity of Alzheimer's disease $A \beta$ peptides is induced by small changes in the A 42 to A 40 ratio. EMBO J. 29, 3408-3420.

Lee, H., Shamy, G.A., Elkabetz, Y., Schofield, C.M., Harrsion, N.L., Panagiotakos, G., Socci, N.D., Tabar, V., and Studer, L. (2007). Directed differentiation and transplantation of human embryonic stem cell-derived motoneurons. Stem Cells 25, 1931-1939.

Li, X.-J., Hu, B.-Y., Jones, S.A., Zhang, Y.-S., Lavaute, T., Du, Z.-W., and Zhang, S.-C. (2008). Directed differentiation of ventral spinal progenitors and motor neurons from human embryonic stem cells by small molecules. Stem Cells 26, 886-893.

Liao, M.-C., Muratore, C.R., Gierahn, T.M., Sullivan, S.E., Srikanth, P., De Jager, P.L., Love, J.C., and Young-Pearse, T.L. (2016). Singlecell detection of secreted A $\beta$ and SAPP $\alpha$ from human IPSC-derived neurons and astrocytes. J. Neurosci. 36, 1730-1746.

McDonald, J.M., Cairns, N.J., Taylor-Reinwald, L., Holtzman, D., and Walsh, D.M. (2012). The levels of water-soluble and triton-soluble $\mathrm{A} \beta$ are increased in Alzheimer's disease brain. Brain Res. 1450, 138-147.

McGowan, E., Pickford, F., Kim, J., Onstead, L., Eriksen, J., Yu, C., Skipper, L., Murphy, M.P., Beard, J., Das, P., et al. (2005). Abeta42 is essential for parenchymal and vascular amyloid deposition in mice. Neuron 47, 191-199.

Moore, S., Evans, L.D.B., Andersson, T., Portelius, E., Smith, J., Dias, T.B., Saurat, N., McGlade, A., Kirwan, P., Blennow, K., et al. (2015). APP metabolism regulates tau proteostasis in human cerebral cortex neurons. Cell Rep. 11, 689-696.

Muratore, C.R., Rice, H.C., Srikanth, P., Callahan, D.G., Shin, T., Benjamin, L.N.P., Walsh, D.M., Selkoe, D.J., and Young-Pearse, T.L. (2014a). The familial Alzheimer's disease APPV717I mutation alters APP processing and Tau expression in iPSC-derived neurons. Hum. Mol. Genet. 13, 3523-3536.

Muratore, C.R., Srikanth, P., Callahan, D.G., and Young-Pearse, T.L. (2014b). Comparison and optimization of hiPSC forebrain cortical differentiation protocols. PLoS One 9, e105807.

Portelius, E., Bogdanovic, N., Gustavsson, M.K., Volkmann, I., Brinkmalm, G., Zetterberg, H., Winblad, B., and Blennow, K. (2010). Mass spectrometric characterization of brain amyloid beta isoform signatures in familial and sporadic Alzheimer's disease. Acta Neuropathol. 120, 185-193.

Scheuner, D., Eckman, C., Jensen, M., Song, X., Citron, M., Suzuki, N., Bird, T.D., Hardy, J., Hutton, M., Kukull, W., et al. (1996). Secreted amyloid beta-protein similar to that in the senile plaques of Alzheimer's disease is increased in vivo by the presenilin 1 and 2 and APP mutations linked to familial Alzheimer's disease. Nat. Med. 2, 864-870.

Serneels, L., Dejaegere, T., Craessaerts, K., Horré, K., Jorissen, E., Tousseyn, T., Hébert, S., Coolen, M., Martens, G., Zwijsen, A., et al. (2005). Differential contribution of the three Aph1 genes to gamma-secretase activity in vivo. Proc. Natl. Acad. Sci. USA 102, 1719-1724. 
Serneels, L., Van Biervliet, J., Craessaerts, K., Dejaegere, T., Horré, K., Van Houtvin, T., Esselmann, H., Paul, S., Schäfer, M.K., Berezovska, O., et al. (2009). gamma-Secretase heterogeneity in the Aph1 subunit: relevance for Alzheimer's disease. Science 324, 639-642.

Shankar, G.M., Li, S., Mehta, T.H., Garcia-Munoz, A., Shepardson, N.E., Smith, I., Brett, F.M., Farrell, M.A., Rowan, M.J., Lemere, C.A., et al. (2008). Amyloid $\beta$-protein dimers isolated directly from Alzheimer brains impair synaptic plasticity and memory. Nat. Med.

\section{4, 837-842.}

Shinohara, M., Fujioka, S., Murray, M.E., Wojtas, A., Baker, M., Rovelet-Lecrux, A., Rademakers, R., Das, P., Parisi, J.E., Graff-Radford, N.R., et al. (2014). Regional distribution of synaptic markers and APP correlate with distinct clinicopathological features in sporadic and familial Alzheimer's disease. Brain J. Neurol. 137, 1533-1549.
Szaruga, M., Veugelen, S., Benurwar, M., Lismont, S., SepulvedaFalla, D., Lleo, A., Ryan, N.S., Lashley, T., Fox, N.C., Murayama, S., et al. (2015). Qualitative changes in human $\gamma$-secretase underlie familial Alzheimer's disease. J. Exp. Med. 212, 2003-2013.

Zeng, H., Guo, M., Martins-Taylor, K., Wang, X., Zhang, Z., Park, J.W., Zhan, S., Kronenberg, M.S., Lichtler, A., Liu, H.-X., et al. (2010). Specification of region-specific neurons including forebrain glutamatergic neurons from human induced pluripotent stem cells. PLoS One 5, e11853.

Zhang, Y., Pak, C., Han, Y., Ahlenius, H., Zhang, Z., Chanda, S., Marro, S., Patzke, C., Acuna, C., Covy, J., et al. (2013). Rapid single-step induction of functional neurons from human pluripotent stem cells. Neuron 78, 785-798. 